\title{
PROGRESS IN MULTI-DIMENSIONAL UPWIND DIFFERENCING
}

\author{
Bram van Leer ${ }^{1}$ \\ Department of Aerospace Engineering \\ University of Michigan \\ Ann Arbor, MI
}

\begin{abstract}
Multi-dimensional upwind-differencing schemes for the Euler equations are reviewed. On the basis of the first-order upwind scheme for a one-dimensional convection equation the two approaches to upwind differencing are discussed: the fluctuation approach and the finitevolume approach. The usual extension of the finite-volume method to the multi-dimensional Euler equations is not entirely satisfactory, because the direction of wave propagation is always assumed to be normal to the cell faces. This leads to smearing of shock and shear waves when these are not grid-aligned. Multi-directional methods, in which upwind-biased fluxes are computed in a frame aligned with a dominant wave, overcome this problem, but at the expense of robustness. The same is true for the schemes incorporating a multidimensional wave model not based on multi-dimensional data but on an "educated guess" of what they could be.

The fluctuation approach offers the best possibilities for the development of genuinely multi-dimensional upwind schemes. Three building blocks are needed for such schemes: a wave model, a way to achieve conservation, and a compact convection scheme. Recent advances in each of these components are discussed; putting them all together is the present focus of a worldwide research effort. Some numerical results are presented, illustrating the potential of the new multi-dimensional schemes.
\end{abstract}

\footnotetext{
${ }^{1}$ This research was supported by the National Aeronautics and Space Administration under NASA Contract Nos. NAS1-18605 and NAS1-19480 while the author was in residence at the Institute for Computer Applications in Science and Engineering (ICASE), NASA Langley Research Center, Hampton, VA 23665.
} 
- 


\section{Introduction}

CFD algorithms for the coming generation of massively parallel computers will have to be extremely robust. They will most likely be implemented on adaptive unstructured grids, and will be used for ambitious simulations of steady and unsteady threedimensional flows. In such a complex environment there is little place left for handtuning parameters that regulate accuracy, stability and convergence of the computations. A typical algorithm will make very intensive use of local data, with a minimum of message passing.

Algorithms of this nature exist already in CFD: they are the upwind-differencing schemes, computationally intensive but unsurpassed in their combination of accuracy and robustness. While these favorable properties are explainable for one-dimensional methods, it is a stroke of luck that upwind schemes work as well as they do for two- and three-dimensional flow. Their design is commonly based on one-dimensional physics, namely, the solution of the one-dimensional Riemann problem that describes the interaction of two fluid cells by finite-amplitude waves moving normal to their interface. The inadequacy of this technique clearly shows up when the numerical solution contains shock or shear waves not aligned with the grid, for instance, by a loss of resolution.

The need to incorporate genuinely multi-dimensional physics in upwind algorithms was recognized as early as 1983 by Phil Roe [1]. A study of discrete multi-dimensional wave models by Roe followed in 1985 (ICASE Report 85-18, also [2]), but it took until 1991 [3] before any algorithms based on such wave models became truly successful. Important contributions to this development were made by Herman Deconinck and collaiorators [3,4] at the Von Kármán Institute in Brussels. The new upwind schemes are formulated on unstructured grids with data in the vertices of triangular or tetrahedral cells.

While genuinely multi-dimensional methods were slowly developing, partial successes were booked by putting some multi-dimensional information into the Riemamn solvers used in conventional upwind schemes. In particular, it became the fashion to obtain a plausible wave-propagation angle from the data, rather than accepting the angle dictated by the grid geometry. The earliest work of this kind is due to Steve Davis [5]; it recently was picked up by a number of authors: Levy, Powell and Van Leer [6], [7], Dadone and Grossman [8, 9], Obayashi and Goorjian [10], Tamura and Fujii [11]. Roughly speaking, they apply Riemann solvers in several, physically appealing, directions; I shall refer to their work as the multi-directional approach.

Related, but closer to the genuinely multi-dimensional approach is the work of Rumsey, Van Leer and Roe $[12,13,14,15]$ and Parpia and Michalek $[16,17]$. These authors independently developed almost identical multi-dimensional wave models based on minimizing wave strengths. These wave models requires only two input states, just 

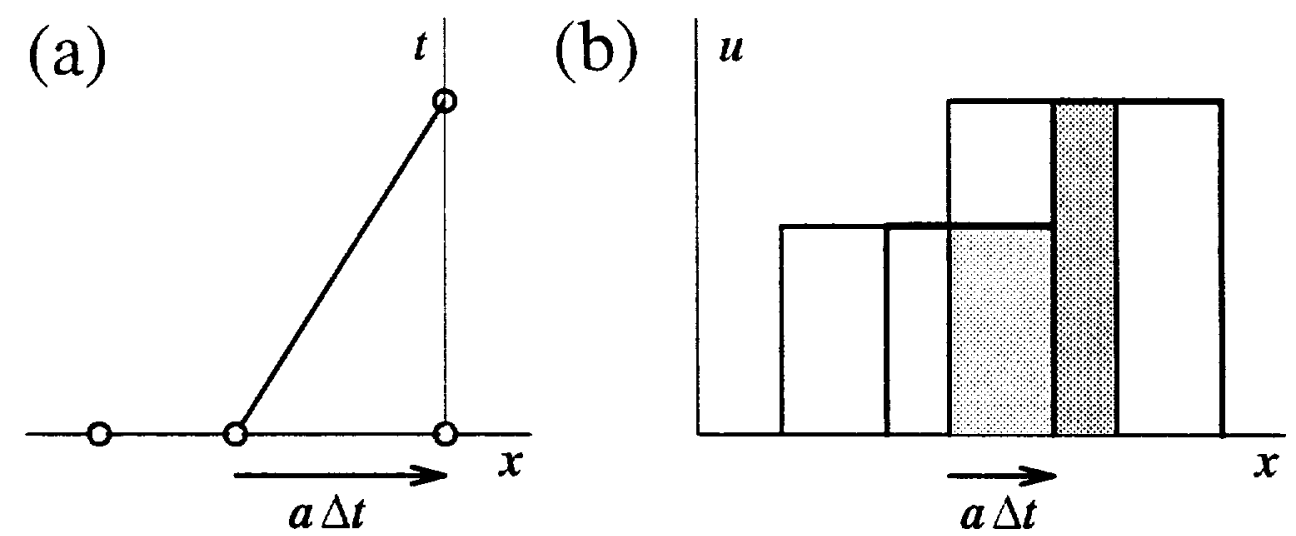

Figure 1: Two views on scalar upwind differencing: (a) nodal-point interpretation; (b) finite-volume interpretation.

as a regular Riemann solver.

In support of these quasi-multi-dimensional approaches, aimed at putting better physics into interface fluxes, some authors have dedicated efforts to improving the interpolation or reconstruction step that precedes the flux calculation. On a structured grid the reconstruction of a non-oscillatory distribution of flow variables from their cell-averages usually is done dimension by dimension; a fully multi-dimensional reconstrucion is indispensable in achieving higher accuracy. Barth and Frederickson [18] indicated how to reconstruct a smooth function up to arbitrarily high order on an unstructured triangulation; Abgrall [19] showed how to implement truly multidimensional limiting of higher derivatives.

In this lecture I shall review a decade of efforts toward multi-dimensional upwinddifferencing, with the accent on the very latest developments. The discussion is limited to the multi-dimensional physics that goes into these methods; multi-dimensional reconstruction will not be further mentioned. For a somewhat different emphasis or point of view the reader is referred to three excellent other reviews of multidimensional methods $[20,21,4]$ that have been presented in the past year.

\section{Two views of one-dimensional upwinding}

In order to appreciate the problems surrounding multi-dimensional upwinding it is useful to consider the principles of one-dimensional upwinding. The reader is assumed to be familiar with the theory of conservative upwind schemes; as a tutorial Roe's [22] review article is recommended.

Upwind differencing is a way of differencing convection terms. For the scalar convection equation

$$
u_{t}+c u_{x}=0
$$

the simplest upwind-difference scheme, of first-order accuracy, reads

$$
\frac{u_{i}^{n+1}-u_{i}^{n}}{\Delta t}+c \frac{u_{i}^{n}-u_{i-1}^{n}}{\Delta x}=0, \quad c \geq 0
$$




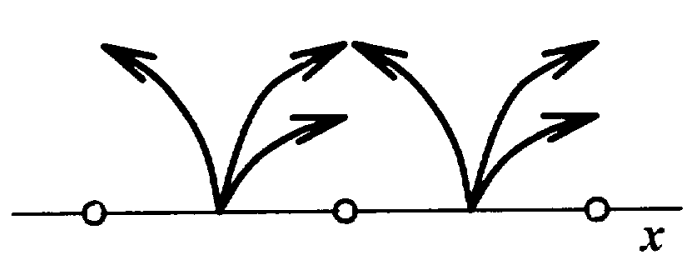

(a)

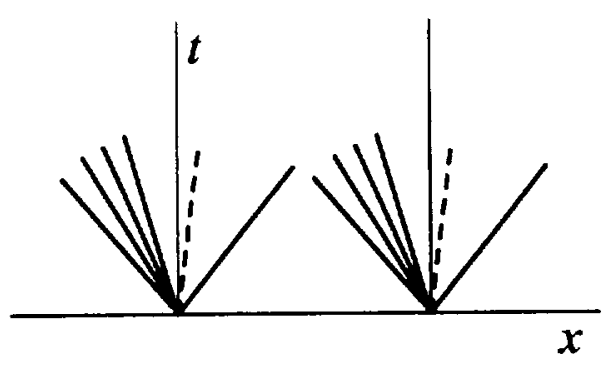

(b)

Figure 2: Two approaches to upwind differencing for the Euler equations: (a) fluctuation approach; (b) finite-volume approach.

$$
\frac{u_{i}^{n+1}-u_{i}^{n}}{\Delta t}+c \frac{u_{i+1}^{n}-u_{i}^{n}}{\Delta x}=0, \quad c<0
$$

Scheme $(2,3)$ can be regarded as a formula for updating, from $t^{n}$ to $t^{n+1}$, either the nodal-point value of $u$ in $x_{i}$, or the cell average of $u$ in cell $i$. These two view-points are illustrated in Figures $1 \mathrm{a}$ and $1 \mathrm{~b}$. The distinction is significant, because it leads to distinct methods for more complex equations. In the development of schemes for the one-dimensional Euler equations, the first view-point has led to the concept of fluctuation splitting, due to Roe [23, 22]; the second view-point is that of Godunov [24] and has led to the projection/evolution or reconstruction/evolution concept of finite-volume schemes, due to Van Leer $[25,26,27]$. Below I shall review the formulas pertinent to each approach.

\subsection{Fluctuation splitting}

Assume the system

$$
U_{t}+F(U)_{x}=0
$$

represents the Euler equations in conservation form, i.e., $U=(\rho, \rho u, \rho E)^{T}$ is the vector of conserved state quantities and $F(U)=\left(\rho u, \rho u^{2}+p, \rho u H\right)^{T}$ is the vector of their fluxes. The equation shows that any local imbalance of the fluxes causes the local solution to change in time. Such a local imbalance is called a fluctuation by Roe $[28,1]$. If source terms are present, their value must be included in the fluctuation $[22]$.

Define the matrix $A(U)$ as the derivative of $F(U)$ with respect to $U$, so that

$$
d F(U)=A(U) d U
$$

It is essential for the technique of fluctuation splitting that this differential relation be replaced by an exact finite-difference analogue, namely,

$$
\Delta F=\hat{A} \Delta U
$$

where $\Delta$ indicates a difference between neighboring nodal points. Roe [29] has indicated how to construct a mean value $\hat{A}$ of $A$ such that Eq. (6) holds exactly for arbitrary pairs of state vectors. For a calorically perfect gas a suitable mean value 
ran easily be obtained by introducing the the parameter vector $w \equiv \sqrt{\rho}(1, u, H)^{T}$. Since both $V(w)$ and $F(w)$ are quadratic in the components of $w$, it follows that Eq. (6) is satisfied by $\hat{A} \equiv A(U(\bar{w}))$, where $\bar{w}$ is the algebraic average of $w$.

Fluctuation splitting requires that the matrix $\hat{A}$ be split into its positive and negative parts, i.e.,

$$
\hat{A}=\hat{A}^{+}+\hat{A}^{-}
$$

so that

$$
\Delta F=\hat{A}^{+} \Delta U+\hat{A}^{-} \Delta U \text {. }
$$

A popular name for this procedure is "flux-difference splitting"; the term "fluctuation splitting" is preferable because it includes source-term splitting. The first term on the right-hand side combines disturbances that propagate forward; in consequence, this term is used to update the right nodal point. The second term combines backwardmoving disturbances and is used to update the left nodal point. This concept is illustrated in Figure 2a. Conservation is ensured because the two terms add up to a perfect flux difference. The first-order update formula becomes

$$
U_{i}^{n+1}=U_{i}^{n}-\frac{\Delta x}{\Delta t}\left(\left(\hat{A}^{+} \Delta U\right)_{i-\frac{1}{2}}+\left(\hat{A}^{-} \Delta U\right)_{i+\frac{1}{2}}\right) .
$$

In practice it often pays to abandon the matrix notation and expand $\Delta U$ and $\Delta F$ in terms of the individual disturbances. This yields

$$
\begin{aligned}
& \Delta U=\sum_{k=1}^{3} \alpha_{k} R_{k}, \\
& \Delta F=\sum_{k=1}^{3} \lambda_{k} \alpha_{k} R_{k},
\end{aligned}
$$

where $\lambda_{k}$ is an eigenvalue of $\hat{A}, R_{k}$ is the corresponding eigenvector, and $\alpha_{k}$ is the wave strength; note that Eqs. (6) and (10) imply Eq. (11). By considering that each fluctuation may move forward or backward through the grid, we recover the splitting formula $(8)$ :

$$
\begin{aligned}
\Delta F & =\sum_{\lambda_{k}<0} \lambda_{k} \alpha_{k} R_{k}+\sum_{\lambda_{k}>0} \lambda_{k} \alpha_{k} R_{k} \\
& \equiv \sum_{k=1}^{3} \lambda_{k}^{+} \alpha_{k} R_{k}+\sum_{k=1}^{3} \lambda_{k}^{-} \alpha_{k} R_{k} \\
& =\hat{A}^{+} \Delta U+\hat{A}^{-} \Delta U .
\end{aligned}
$$

\subsection{Finite-volume approach}

In the finite-volume approach the focus is on the numerical flux function $F\left(U_{L}, U_{R}\right)$, a recipe for computing the interface fluxes from the states $U_{L}$ and $U_{R}$ on the left and right sides of the interface. The generic formula for updating cell averages of the conserved quantities is

$$
U_{i}^{n+1}=U_{i}^{n}-\frac{\Delta t}{\Delta x}\left(F_{i+\frac{1}{2}}^{n}-F_{i-\frac{1}{2}}^{n}\right)
$$


In Godunov's first-order scheme the interface Hux is taken from the solution at $t>0$ of Riemann's initial-value problem with input data

$$
\begin{aligned}
& U(x, 0)=U_{L}, \quad x>0, \\
& U(x, 0)=U_{R}, \quad x<0
\end{aligned}
$$

this is illustrated in Figure $2 \mathrm{~b}$.

For many applications it is not necessary to use the exact solution to this problem, hence the activity in the research area of "approximate Riemann solvers" $[2: 3,30,31]$. Adopting Roe's [23] approximate solution, which is the exact solution of the locally linearized equation

$$
U_{t}+\hat{A} U_{x}=0
$$

we find three equivalent formulas for the interface flux:

$$
\begin{aligned}
& F\left(U_{L}, U_{R}\right)=F_{L}+\hat{A}^{-} \Delta U, \\
& F\left(U_{L}, U_{R}\right)=F_{R}-\hat{A}^{+} \Delta U, \\
& F\left(U_{L}, U_{R}\right)=\frac{1}{2}\left(F_{L}+F_{R}\right)-|\hat{A}| \Delta U,
\end{aligned}
$$

where

$$
|\hat{A}|=\hat{A}^{+}-\hat{A}^{-} \text {. }
$$

In practice the formula (19) is preferred because of its symmetry; the expanded form is

$$
F\left(U_{L}, U_{R}\right)=\frac{1}{2}\left(F_{L}+F_{R}\right)-\frac{1}{2} \sum_{k=1}^{3}\left|\lambda_{k}\right| \alpha_{k} R_{k} .
$$

Inserting the flux (19) into the finite-volume scheme (13) yields an scheme that, with the help of the identity (6), reduces precisely to the fluctuation scheme (9). Yet, there exists an important difference between Eqs. $(19,13)$ and Eq. $(9)$ : in the latter the matrix $\hat{A}$ must satisfy the identity $(6)$ in order to maintain conservation, while in Eq. (19) the matrix $|\hat{\hat{A}}|$ may be derived from any average $\hat{A}$ without endangering conservation. The flux formula (19), due to Van Leer [32, 33], preceded the fluctuation approach of Roe [2:3], based on (6), by a decade.

\section{Intermezzo: how good is one-dimensional up- winding?}

To appreciate the superior accuracy and robustness of upwind differencing in one dimension, consider the numerical results shown in Figure 3 and 4, taken from [34] and [35], respectively. In Figure 3 a the exact and discrete Mach-number distributions for choked flow through a converging-diverging channel are superimposed. Firstorder fluctuation splitting was used, including source-term splitting $[22,36]$ and a special splitting near the sonic point [34]. Although the update formula is only firstorder accurate, it can be shown that the scheme yields second-order accurate steady solutions. In fact, in the steady state the scheme reduces to the two-point box scheme on all meshes except near a sonic point and inside a shock structure, where it becomes a three-point scheme. This yields the smooth transition through the sonic point and 
the crisp shock transition in the displayed results. Figure $3 \mathrm{~b}$ shows the residualconvergence histories for three increasingly powerfull marching techniques: global time-stepping, local time-stepping and characteristic time-stepping [35]; these look uneventful. In Figure 4a a shockless transonic solution is reached from initial values containing 7 shocks and 8 sonic points; again, the residual-convergence history in Figure $4 \mathrm{~b}$ for local time-stepping shows nothing unusual.

It is this type of performance we wish to preserve when extending upwind differencing to higher dimensions.

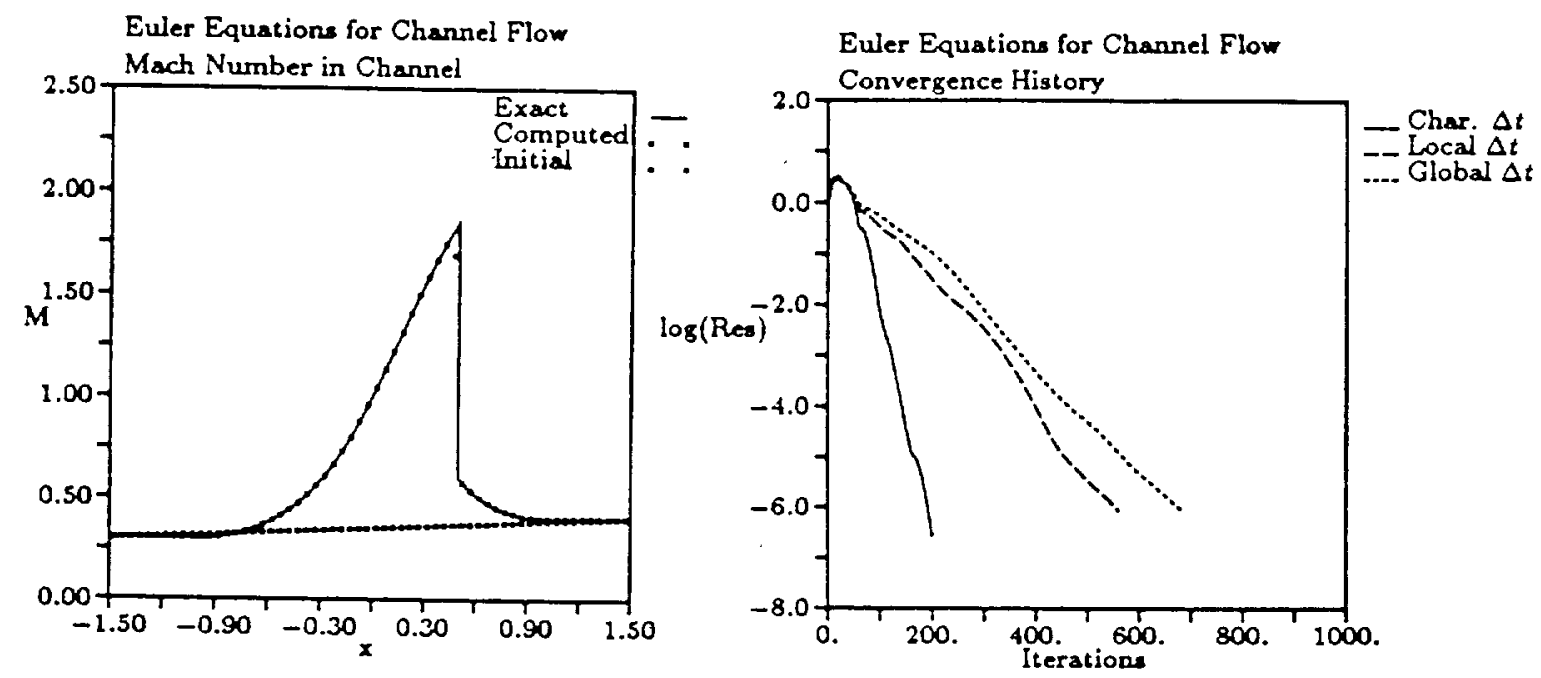

Figure 3: Choked flow through a converging-diverging chamel, computed with a fluctuation scheme. (a) Initial and final Mach-number distributions; (b) residualconvergence histories for global, local and characteristic time-stepping.
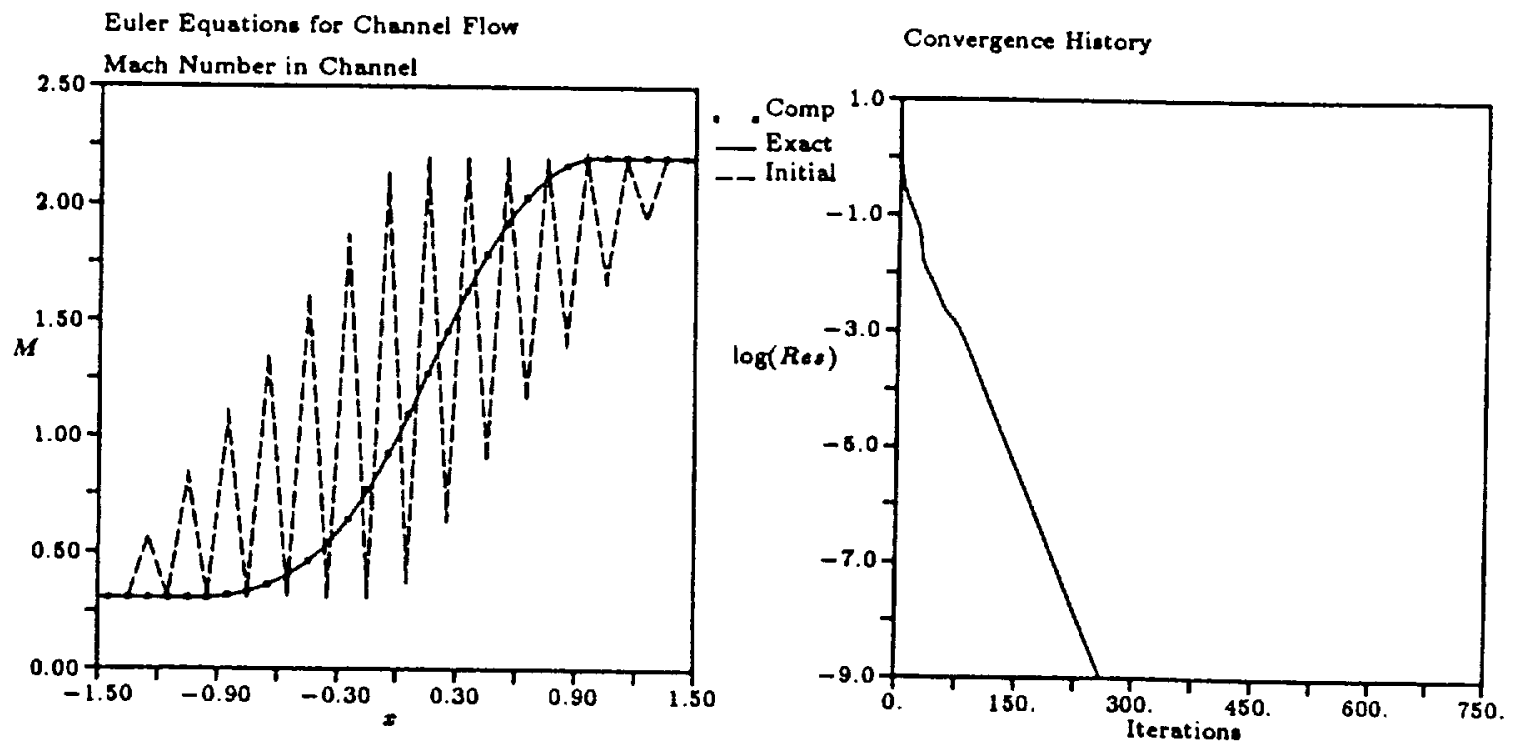

Figure 4: Transonic flow in a converging-diverging channel, computed with a fluctuation scheme. (a) Initial and final Mach-number distributions; (b) residual-convergence history for local time-stepping. 


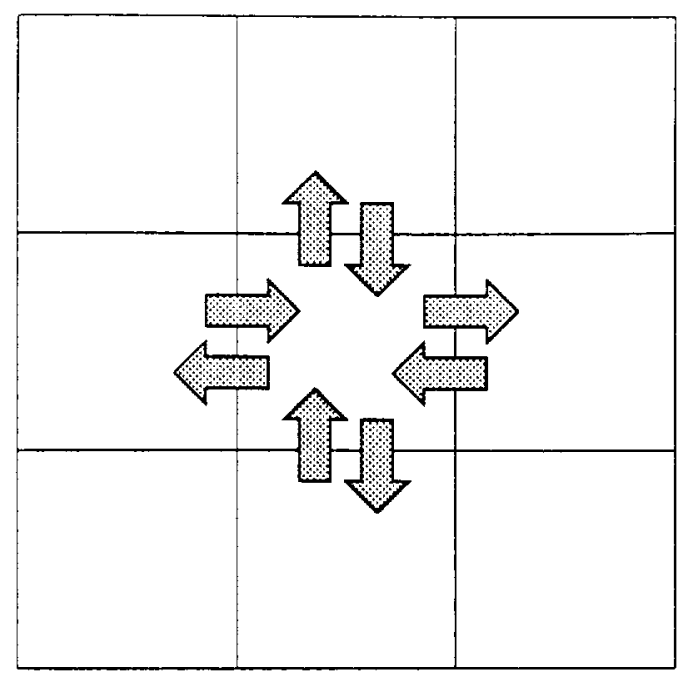

Figure 5: Extending the finite-volume method to two dimensions by solving onedimensional Riemann problems at all cell faces. The arrows symbolize the exchange of information between cells in the direction normal to their interface.

\section{Multi-dimensional extension of the finite-volume method}

The standard way to extend upwind differencing to the multi-dimensional Euler equations is still the same as indicated by Godunov et al. [37] in 1961. For first-order accuracy, initial values are assumed to be constant in each cell, just as in one dimension; fluxes at cell interfaces again follow from solving one-dimensional Riemann problems of the type $(14,15)$, with $x$ now measuring distance along the normal to the interface. This is illustrated by Figure 5.

It is the projection of the true initial values onto cellwise constant distributions (or linear $[25,26]$ or quadratic $[25,38,39]$ or even higher-order distributions [40]) that creates discontinuities at the interfaces. This leads us to introducing plane wave fronts parallel to the interface, and selecting, out of all possible directions, the interface normal as the direction for wave propagation. If the solution contains only shock and/or shear waves aligned or nearly aligned with the grid, this choice happens to be the correct one, and high resolution of such waves can be achieved in the steady state, just as in one dimension. If, however, such waves are far from aligned with the grid, they get misrepresented by the upwind scheme as pairs of grid-aligned waves, as shown in Figure 6 for a shear wave. Thus, a grid-oblique stationary wave may be represented by several grid-aligned rumning waves, leading to higher numerical dissipation and a considerable loss of resolution.

Another purely numerical artifact caused by grid-aligned upwinding is the presence of pressure disturbances across a grid-oblique shear layer. First observed by Venkatakrishnan [41], the explanation was provided by Rumsey et al. [12]; this phenomenon is further discussed in Section 4.2.

From the above critique one should not conclude that in higher dimensions the standard upwind methods are inferior to other methods; the loss of accuracy just is much more obvious for upwind methods. 

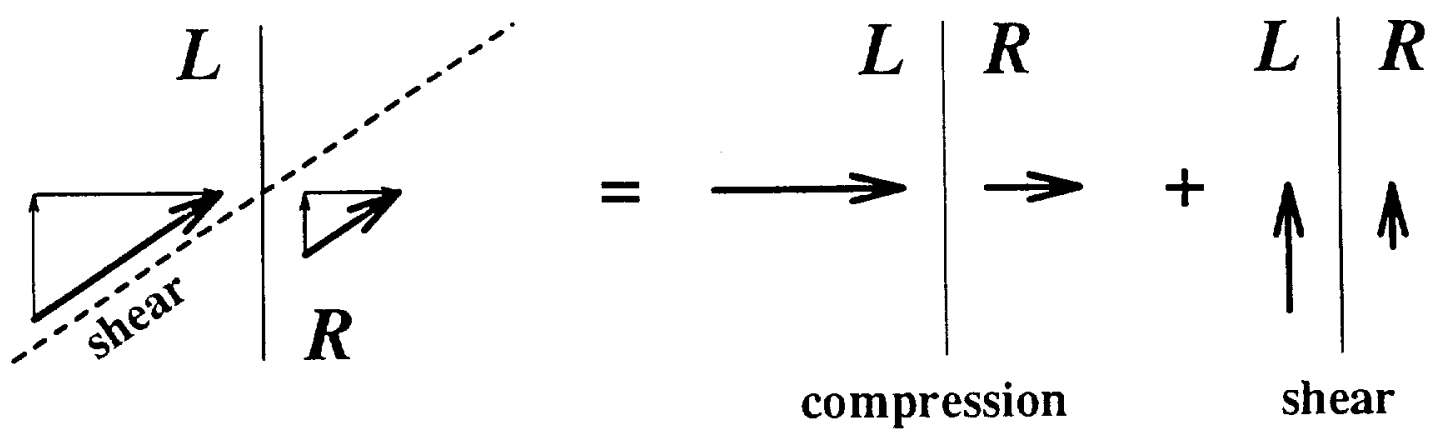

Figure 6: Misinterpretation of a grid-oblique shear wave by grid-aligned upwinding.

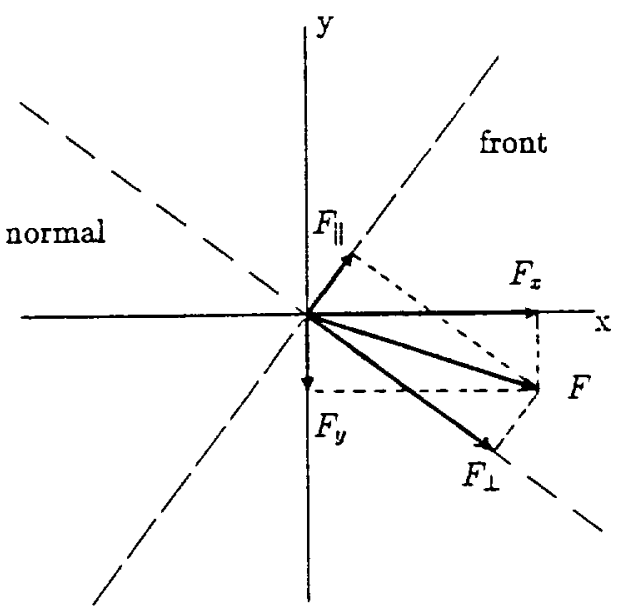

Figure 7: Fluxes in a frame aligned with a wave front oblique to the grid lines.

\subsection{Multi-directional methods}

The smearing of oblique shock waves in numerical solutions has received considerable attention, and a proportionally large research effort has been spent in mending this weakness. The prevailing idea is to solve the Riemann problem in a direction more appropriate than the grid direction. One immediate consequence of leaving the gridaligned frame is that solving one Riemann problem no longer suffices. Figure 7 shows that, in two dimensions, both flux vectors in the rotated frame are needed for the construction of the fluxes normal to the interface.

Consider, for example, Figure 8, showing a rotated coordinate system aligned with level lines representing a shock front in a discrete solution. It makes sense to solve a one-dimensional Riemann problem in the direction normal to the front, i.e. using the flow-velocity components in that direction; this yields the flux in the normal direction. The input states for the Riemann solver are $U_{L \perp}=U_{L}$ and $U_{R \perp}=U_{R}$. The flux tangential to the shock should be obtained from state values located at $L_{\|}$and $R_{\|}$; using $U_{L}$ and $U_{R}$ once more would completely destroy the effect of the rotation $[7,14])$. These values could be approximated by

$$
U_{L \|}=U_{R \|}=\frac{1}{2}\left(U_{L}+U_{R}\right)
$$

this, however, implies central differencing along the shock and leads to odd-even decoupling in that direction $[6,7,42]$. 


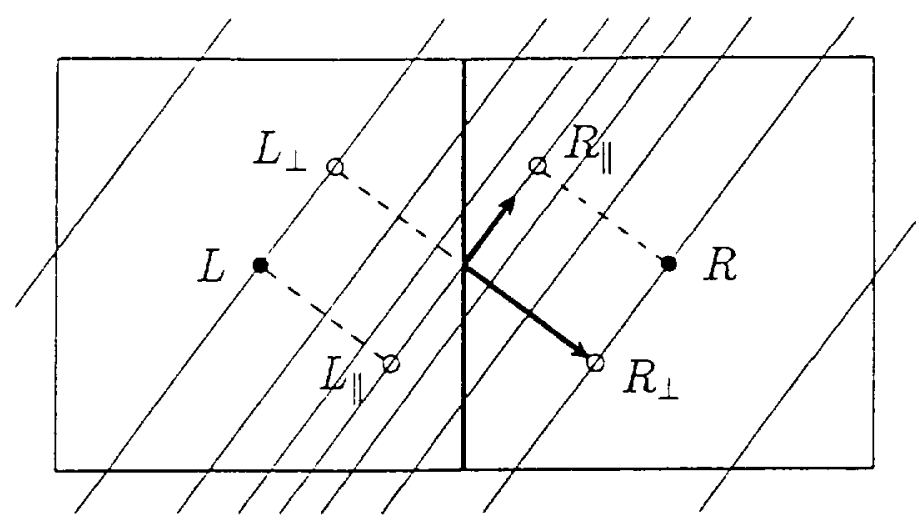

Figure 8: A simple multi-directional flux formula.

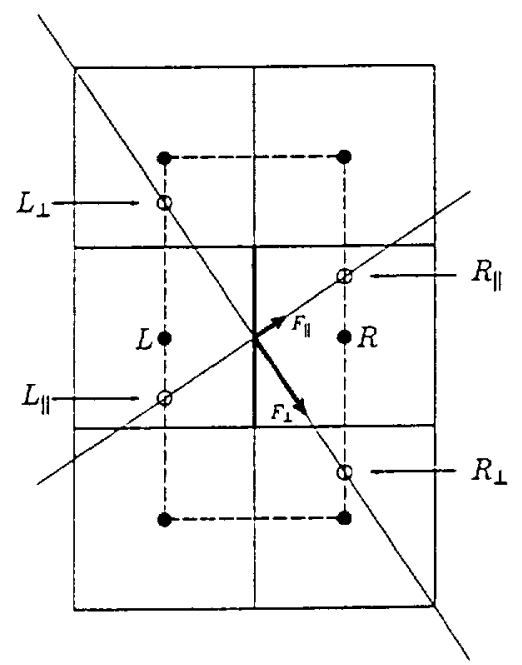

Figure 9: Input states for the Riemann problems in the flux computation according to Levy et al.

In the work of Davis [5, 43], dating back as far as 1983, the computation of the tangent flux actually is more complicated than that of the normal flux. The more recent work of Levy et al. $[6,7,42]$ and Dadone and Grossman $[8,9]$ is more mature in that the fluxes are reated without distinction. Figure 9 shows how pairs of input states to the two Riemann problems, $\left(U_{L \perp}, U_{R \perp}\right)$ and $\left(U_{L \|}, U_{R \|}\right)$, are selected according to Levy et al. In their first-order method the input states in the rotated frame are obtained by linear interpolation between neighboring states in a ring of cells surrounding the interface; Dadone and Grossman simply take the value in the nearest cell, which apparently adds to the robustness of the method. Another, wider ring of cells is needed for achieving second-order accuracy.

Various choices can be made for the rotation angle of the frame in which the Riemann problems are solved. A sensitive quantity is the direction of the velocitydifference vector, $\vec{V}_{R}-\vec{V}_{L}$, which was adopted by Davis and also is crucial to the approach of Rumsey and Parpia (see Section 4.2). Levy et al. use the direction of the velocity-magnitude gradient $\vec{\nabla}|\vec{V}|$, which can detect both shock and shear waves, while Dadone and Grossman use the pressure gradient $\vec{\nabla} p$, which only detects shocks. 
For a more detailed description of the multi-directional approach the reader may be referred to reference [9] in these proceedings.

After a decade of multi-directional methods, what benefits have been demonstrated? Surely, these methods yield impressive results when applied to first-order schemes: shock and shear waves not aligned with the grid are represented as if computed with a higher-order method. The improvement brought to higher-order schemes, though, is a lot less spectacular, and this is understandable. On the one hand, there is not much room left for a further reduction of wave spread (more for shear waves than for shock waves); on the other hand, loss of monotonicity may occur, against which there are no effective limiters, and convergence to a steady state suffers under the strong nonlinearity of the methods.

In my opinion, the multi-directional approach has had a clear impact on computational fluid dynamics. Although complete multi-directional methods will survive only if the problem of ensuring robustness can be solved, I expect that elements of such methods may find their way into standard, direction-split codes, to help resolve flow features arising in specific flow problems.

\subsection{Minimum-strength wave models}

In the work of Rumsey, Roe and Van Leer [14] and Parpia and Michalek [17], the orientation of the cell interface is de-emphasized. The spatial discretization is no longer regarded as generating a discontinuity along the interfaces; instead, an attempt is made to find out what waves are actually propagating near the interface. This, of course, requires data spanning a multi-dimensional part of space; if only the two states $U_{L}$ and $U_{R}$ are to be used, a theoretical conjecture must make up for the missing information.

In the basic wave model of Rumsey et al. a special set of 4 waves is used to match the state difference $U_{R}-U_{L}$; for uniqueness, the sum of the wave strengths is minimized. Three of these waves follow from solving a one-dimensional Riemann problem in the direction of the velocity difference $\Delta \vec{V}$, the fourth wave is a shear wave normal to the other three. This choice of waves makes sense from a kinematic point of view, as illustrated by Figure 10. It shows that a velocity difference $\Delta \vec{V}$ can be explained by an acoustic wave traveling in the direction of $\Delta \vec{V}$ as well as a shear wave traveling in the normal direction. Which explanation is the more likely one may be determined by also considering the pressure difference $p_{R}-p_{L}$ : a large value favors the acoustic explanation, while a small value favors the shear explanation. The minimization procedure takes the full state difference $U_{R}-U_{L}$ and comes up with a plausible explanation in terms of all four waves. The method of Parpia and Michalek differs only in the choice of the functional that is minimized. Figure 11 shows the configuration of the plane waves crossing the interface. In practice both methods include a fifth wave, a weak shear wave, which corrects for the difference between the true direction of $\Delta \vec{V}$ and the direction actually used; the latter may have been held over from a previous iteration ("frozen"), for improvement of convergence.

The word "plausible" used above indicates that the minimization procedure only makes an educated guess: it is possible to compose a set of initial values that is totally misinterpreted. Consider, for instance, the head-on collision of two gases that have equal, negligible pressures. In reality two strong shocks are formed, moving into the 


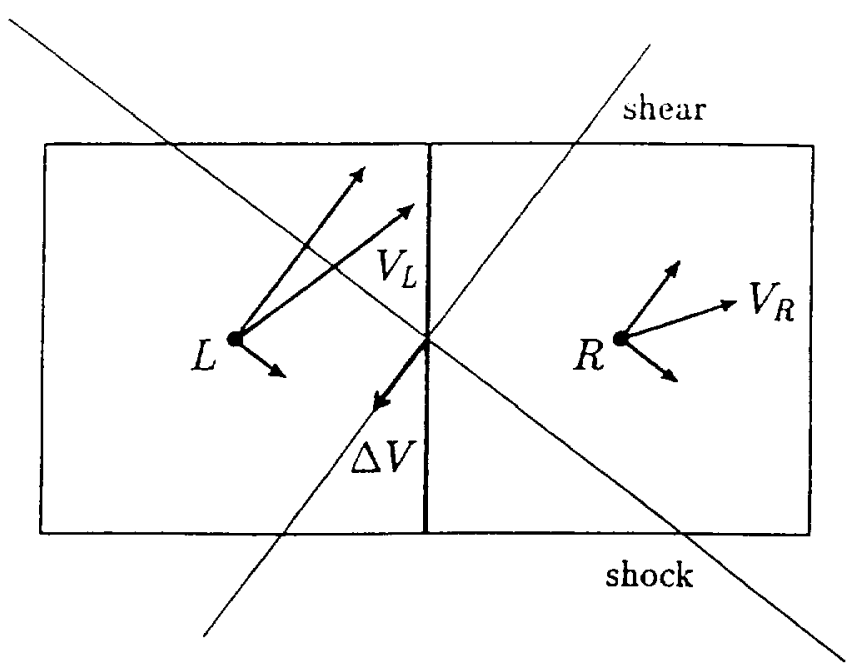

Figure 10: Shock or shear?

gases. The procedure sees as input a velocity difference not accompanied by a pressure difference, hence calls for a single shear wave, as if the gases avoided collision!

The flux formula based on the above wave model is worth some discussion. Assuming the system

$$
U_{t}+F(U)_{x}+G(U)_{y}=0,
$$

with flux Jacobians $A(U)$ and $B(U)$, represents the two-dimensional Euler equations, we may again write $\Delta U$ as a sum:

$$
\Delta U=\sum_{k=1}^{5} \alpha_{k} R_{k}
$$

The vector $R_{k}$ is now an eigenvector of the matrix

$$
\hat{A} \cos \theta_{k}+\hat{B} \sin \theta_{k},
$$

where $\theta_{k}$ indicates the propagation angle of the $k$-th wave; the matrices $\hat{A}$ and $\hat{B}$ are standard Roe-averages. The upwind-biased interface flux is defined by

$$
F\left(U_{L}, U_{R}\right)=\frac{1}{2}\left(F_{L}+F_{R}\right)-\sum_{k=1}^{5}\left|\lambda_{k} \cos \left(\theta_{k}-\theta_{\text {normal }}\right)\right| \alpha_{k} R_{k},
$$

i.e. still by formula (21), but with the wave speeds $\lambda_{k}$ projected onto the interface normal. Although this formula seems trivial, it must be pointed out that there no longer exists a relation between $\Delta F$ and $\Delta U$ like (6).

In numerical practice minimum-strength wave models appear to bring the same benefits and problems as multi-directional methods: great improvements in shock and shear resolution for first-order methods, much smaller improvements for second-order methods, and possible loss of monotonicity and convergence.

To illustrate the performance of this class of methods, consider Figures 12a and 12b. Both show pressure plots for steady viscous flow over a NACA 0012 airfoil at $3^{\circ}$ angle of attack and Reynolds number 5000 , computed on a $129 \times 49$ O-grid by Rumsey $[12,14]$. Under these conditions the flow separates from the upper surface, 


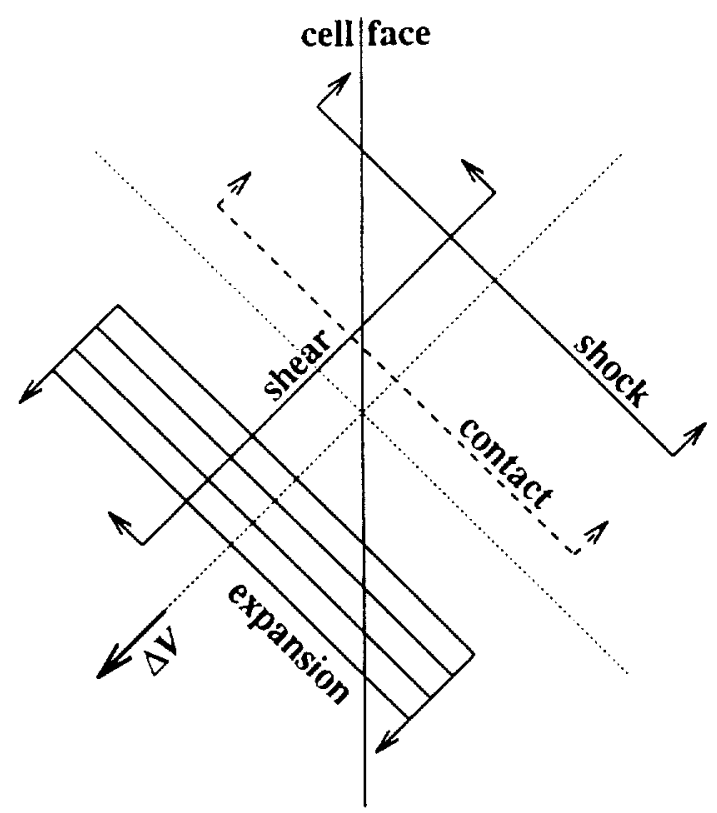

Figure 11: Plane waves crossing a cell face according to the model of Rumsey et al.

producing a detached shear layer oblique to the grid. For the results of Figure 12a a second-order MUSCL-type scheme [26, 44] was used, with Roe's [23] standard gridaligned Riemann solver. The Riemann solver misinterprets the oblique shear as an grid-aligned shear plus an acoustic wave (see Figure 6); the latter causes a pressure rise or drop at the interface. Correspondingly, the steady solution shows pressure fluctuations across the shear layer, so that its presence can actually be detected in pressure plots. A grid-refinement study shows that the disturbances scale with the mesh size. This phenomenon was first observed by Venkatakrishnan [41] and correctly explained by Roe; in fact, it motivated the work of Rumsey, Van Leer and Roe. As seen from Figure $12 \mathrm{~b}$, the minimum-strength wave model properly recognizes the oblique shear layer and generates clean pressure contours.

The same method gives an unexpected improvement in the representation of inviscid stagnating flow. The explanation is found in Figure 13, showing the turning of the flow near a stagnation point $S$ as represented by the discrete velocities in the three cells marked 1,2 and 3.. A grid-aligned Riemann solver interprets the velocity difference between vertical neighbors 1 and 2 as a compression $\left(V_{y 1}>V_{y 2}\right)$, and the velocity difference between horizontal neighbors 2 and 3 as an expansion $\left(V_{x 2}<V_{x 3}\right)$; this leads to pressure variations of the order of $\Delta V$. The wave model detects only very small pressure changes $\left(\Delta p \sim \rho \Delta\left(V^{2}\right)\right)$ and therefore explains both velocity differences by shear waves. Although this still is not the right explanation, the result is a decrease in numerical entropy production. The effect is rather large for first-order methods, as can be judged from Figure 14 showing entropy contours for inviscid flow over a NACA 0012 airfoil at $M=0.3, \alpha=1^{\circ}$, on a sequence of $\mathrm{O}$-grids. The reduced entropy levels lead directly to reduced numerical drag levels, as Figure 15a shows. For second-order schemes the effect, as usual, is less dramatic; the drag values are given in Figure 15b. 
(a)

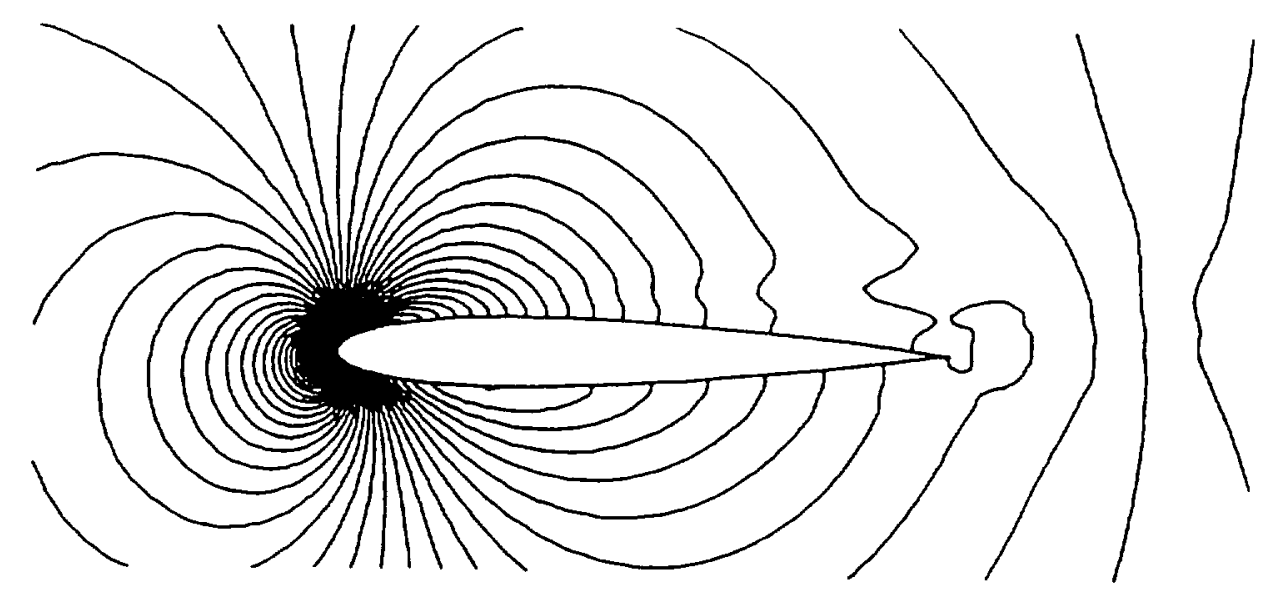

(b)
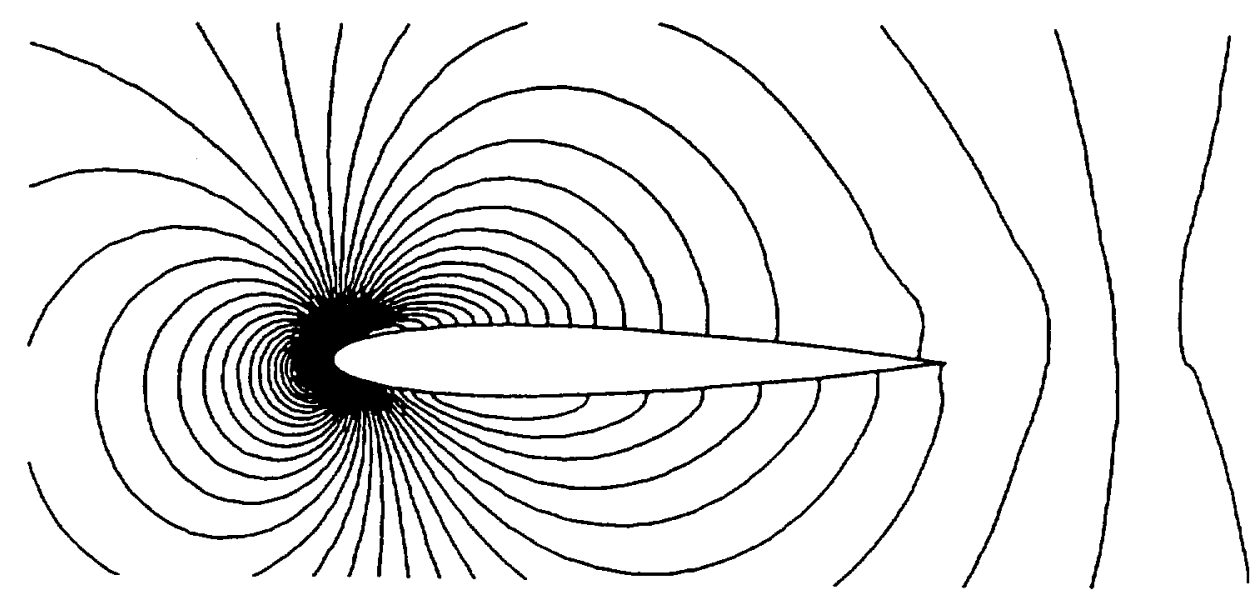

Figure 12: Viscous separating flow over a NACA 0012 airfoil at $M=0.5, \alpha=3^{\circ}$ and $R e=5000$. Pressure contours on a $129 \times 49 \mathrm{C}$-grid, obtained with a second-order upwind scheme incorporating (a) Roe's grid-aligned Riemann solver; (b) the five-wave model of Rumsey et al.

\section{Multi-dimensional fluctuation approach}

The fluctuation approach to upwind differencing lends itself better to extension into higher dimensions than the finite-volume approach. Recall that a fluctuation is a local flux imbalance causing a non-zero time derivative of the local solution. For the one-dimensional Euler equations (4) the quantity $-\Delta F$ equals the residual evaluated on a one-dimensional mesh:

$$
\int_{\text {mesh }} U_{t} d x=-\int_{\text {mesh }} F_{x} d x=-\Delta F .
$$

This suggests extension of the fluctuation approach beyond one dimension by regarding each multi-dimensional mesh residual as the sum of a finite number of waves (say, $m$ ), moving in all possible directions. Thus we discretize the two-dimensional Euler equations as

$$
\iint_{\text {mesh }} U_{t} d x d y=-\oint(F d y-G d x)=\sum_{k=1}^{m} \lambda_{k} \alpha_{k} R_{k}
$$




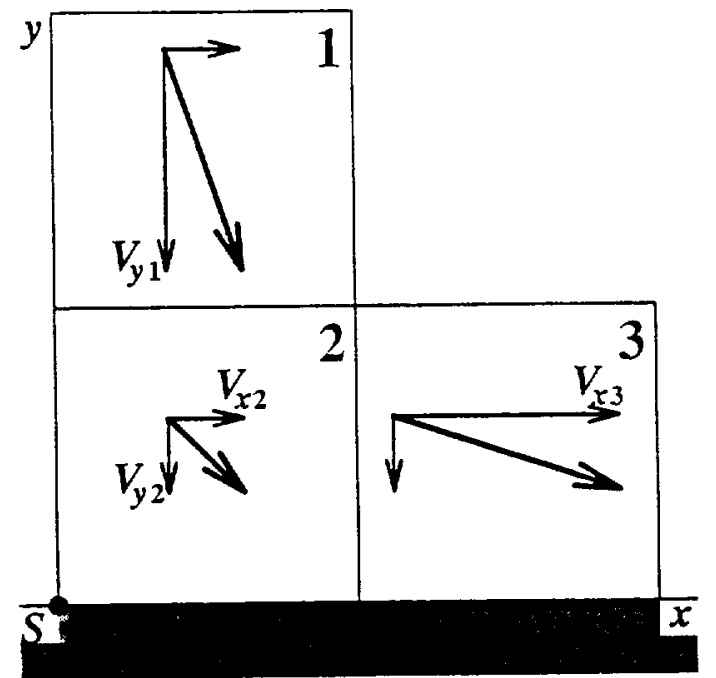

Figure 13: Turning of the flow in three cells near a stagnation point $S$ at a wall.

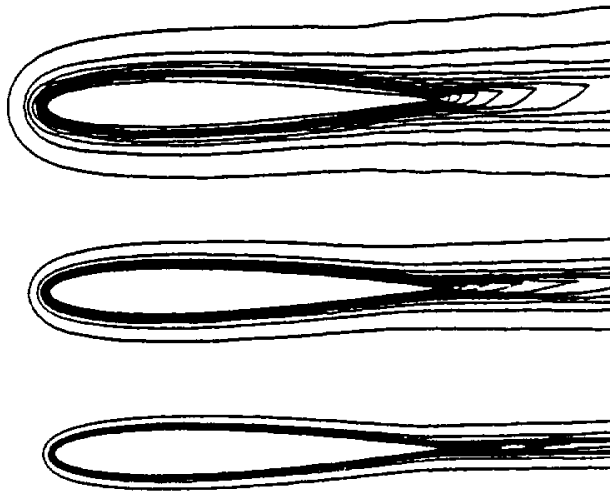

(a)
$65 \times 19$

$129 \times 37$

$257 \times 73$

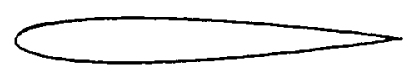

(b)

Figure 14: Entropy contours for inviscid flow over a NACA 0012 airfoil at $M=0.3$, $\alpha=1^{\circ}$, generated on a sequence of $\mathrm{O}$-grids with a first-order scheme incorporating (a) Roe's grid-aligned Riemann solver; (b) the five-wave model of Rumsey et al.

where the matrices $\hat{A}$ and $\hat{B}$ are multi-dimensional averages that remain to be defined. Since the fluctuation approach is a nodal-point approach, and we wish to develop only schemes of maximum compactness, we shall use a grid of triangular meshes, with data given in the nodal points. For the computation of the residual on such meshes it suffices to apply the trapezoidal integration rule on each side of the triangle. The fluctuations resulting from residual decomposition must be sent to the triangle's vertices according to some distribution scheme that approximates the convection equation.

It follows that, for the construction of a genuinely multi-dimensional upwinddifferencing scheme, three components are needed:

1. A reliable multi-dimensional wave model for representing the residual;

2. A way to ensure conservation, i.e. a multi-dimensional extension of Roe's matrix average; 

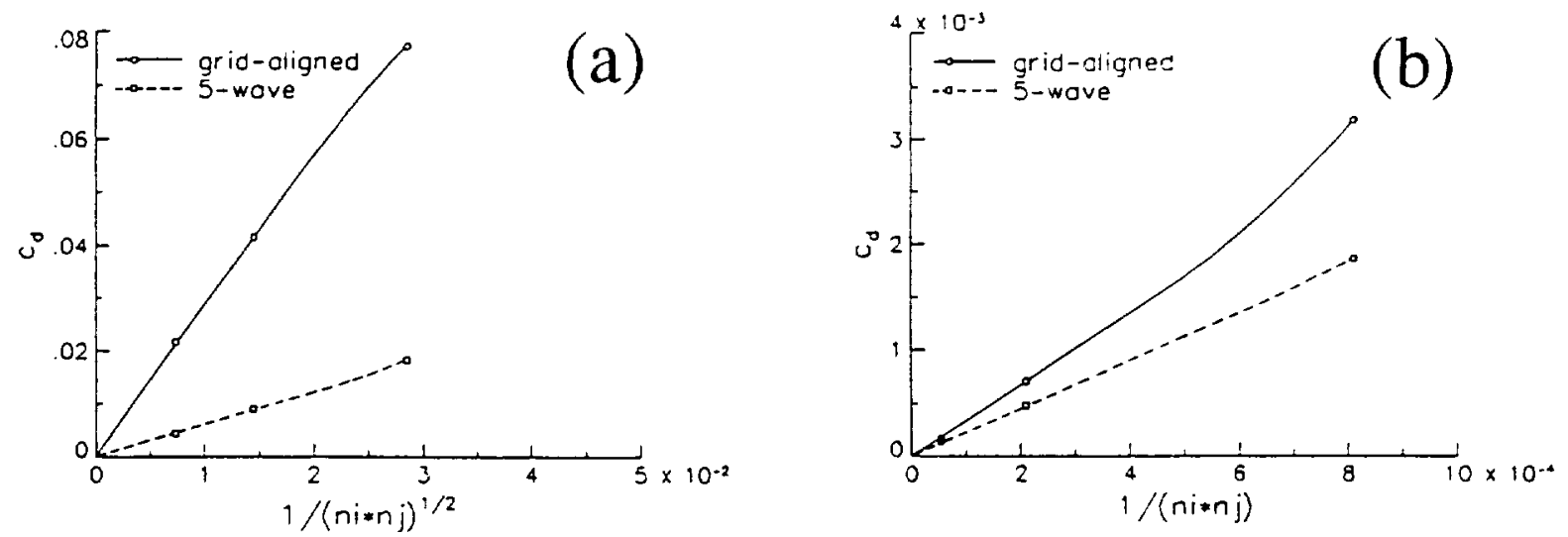

Figure 15: Grid-convergence study of the drag coefficient based on (a) the first-order solutions of Figure 14; (b) the corresponding second-order solutions.

3. A multi-dimensional convection scheme for advancing the waves.

Each of these will be discussed in a separate subsection.

\subsection{Multi-dimensional wave models}

The modeling of a local Euler residual by a finite number of waves was launched as a research subject by Roe [2]; his first paper, however, gave no specific instructions as to how the model would be used in a numerical integration of the Euler equations. This is not surprising, given that the other problems - multi-dimensional conservation and advection - had not yet been addressed.

The latest version of Roe's wave model calls for four acoustic waves, rumning along the principal strain axes of the local fluid element, a shear wave making a $45^{\circ}$ angle with the acoustic waves, and an entropy wave running in the direction of the entropy gradient; see Figure 16. Thus, $m=6$ in Eq. (28). These six waves are defined by two independent angles and six strengths; therefore, eight independent pieces of information need to be supplied per triangular mesh. This information is available in the form of the gradient of the state vector; its mesh value is computed with the trapezoidal rule from the following boundary integrals:

$$
\begin{aligned}
& \widehat{U}_{x} \equiv \frac{1}{\text { Area }} \iint_{\text {mesh }} U_{x} d x d y=\frac{1}{\text { Area }} \oint_{\text {mesh }} U d y ; \\
& \widehat{U}_{y} \equiv \frac{1}{\text { Area }} \iint_{\text {mesh }} U_{y} d x d y=-\frac{1}{\text { Area }} \oint_{\text {mesh }} U d x .
\end{aligned}
$$

A detailed discussion of this wave model, including the three-dimensional case, can be found in Roe's contribution to the present volume [45]; numerical results obtained with this model are presented in the contribution by Catalano et al. [46].

This section would not be complete without a discussion of the work of Hirsch and collaborators [47, 48, 49]. Their multi-dimensional approach is based on diagonalizing the Euler equations, i.e. changing these into a system of convection equations, by a transformation of state variables. The transformation itself depends on the local gradient of the solution, making the diagonalization essentially nonlinear. For certain data the transformation does not exist, in which case it is chosen so as to minimize 


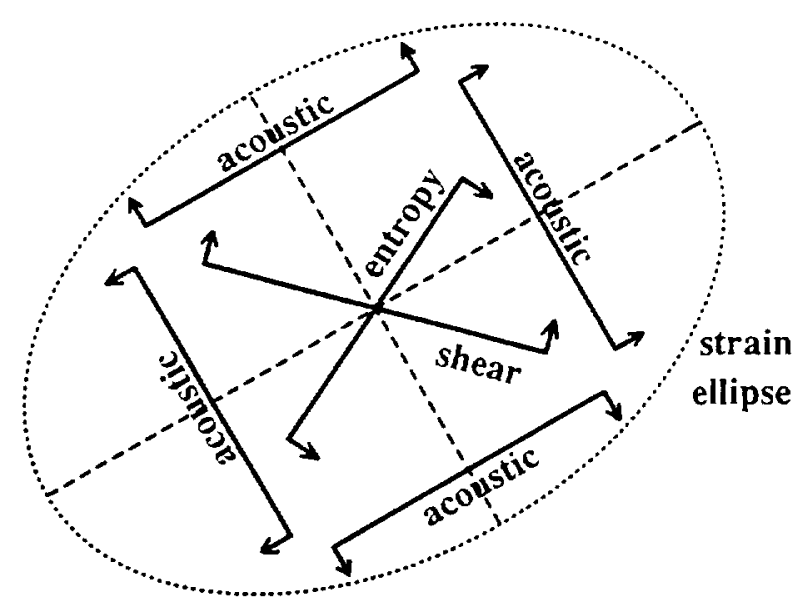

Figure 16: Roe's two-dimensional six-wave model. The acoustic waves run parallel to the principal strain axes (dashed); the strain ellips (dotted) shows the kinematic deformation of a circular fluid element.

the off-diagonal terms. The update scheme, though, can be made identical to a fluctuation-based scheme: decomposition of the residual along certain eigenvectors, followed by convection of the components [50]. In two dimensions the diagonalization is equivalent to using one particular four-wave model; clearly, the fluctuation approach offers much more flexibility.

\subsection{Multi-dimensional conservation}

The multi-dimensional extension of Roe's averaging of the flux Jacobian was independently discovered by Roe and Struijs, and is presented in a joint paper [51]. This very recent (1991) addition to the multi-dimensional toolbox applies exclusively to triangular meshes in two dimensions and tetrahedral meshes in three dimensions. The following description and explanation of the two-dimensional averaging apply to the special case of a calorically perfect gas.

To begin with, assume that the parameter vector $w=\sqrt{\rho}(1, u, v, H)$ is distibuted linearly over a mesh triangle with vertices labeled 1,2 and 3. Denote the average of $w$ over the triangle by $\bar{w}$; we then have

$$
\bar{w}=\frac{1}{3}\left(w_{1}+w_{2}+w_{3}\right) .
$$

As before, $U(w)$ and $F(w)$, and also $G(U)$, are quadratic in the components of $w$, so that the Jacobian matrices $U_{w}, F_{w}$ and $G_{w}$ are linear in $w$, and therefore also in $x$ and $y$. Considering that $U_{x}=U_{w} w_{x}, U_{y}=U_{w} w_{y}$, etc., where $w_{x}$ and $w_{y}$ are constant over the entire triangle, we conclude that $\vec{\nabla} U, \vec{\nabla} F$ and $\vec{\nabla} G$ also vary linearly over the triangle. Using the definition of the mesh-averaged gradient $\widehat{\vec{\nabla} U}$ given in Eqs. (29), (30), and similar definitions of $\widehat{\vec{\nabla} F}$ and $\widehat{\vec{\nabla} G}$, we easily derive the relations,

$$
\begin{aligned}
\widehat{\vec{\nabla} F} & \equiv A(U(\bar{w})) \widehat{\vec{\nabla} U}, \\
\widehat{\vec{\nabla} G} & \equiv B(U(\bar{w})) \widehat{\vec{\nabla} U},
\end{aligned}
$$




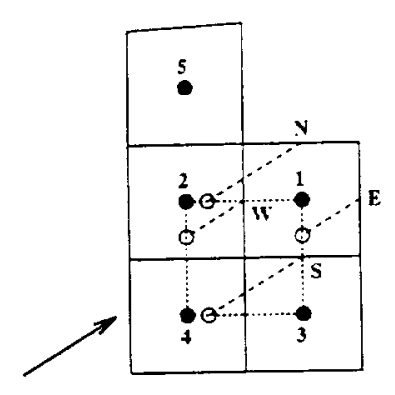

(a)

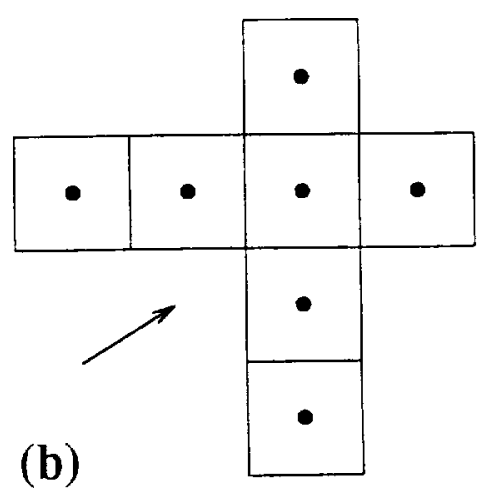

(b)

Figure 17: Stencils of two-dimensional upwind convection schemes; case $a>b>0$. (a) Sidilkover's second-order scheme. The fluxes for cell 1 nominally are compurted by linear interpolation between upstream pairs of data, but the fluxes at the North and South faces must be limited to prevent numerical oscillations. The limiters are based on the ratios $a\left(u_{1}-u_{2}\right) /\left[b\left(u_{5}-u_{2}\right)\right]$ and $a\left(u_{3}-u_{4}\right) /\left[b\left(\mathrm{u}_{2}-u_{4}\right)\right]$, respectively. (b) Standard second- or third-order grid-aligned scheme.

which are direct extensions of the one-dimensional relation (6). The extension to three-dimensional averaging is self-evident.

\subsection{Multi-dimensional convection}

The pursuit of multi-dimensional convection schemes has kept a number of authors busy over the past three years. In two dimensions the basic equation to be solved is

$$
u_{t}+a u_{x}+b u_{y}=0,
$$

where $a$ and $b$ are constant velocity components, or, in vector notation,

$$
u_{t}+\vec{a} \cdot \vec{\nabla} u=0
$$

The first significant work was that of Sidilkover [52], who, among other things, showed how a second-order upwind scheme, with residual computed on a square mesh, can be made non-oscillatory by standard limiters without undue spreading of the stencil. The domain of dependence for this algorithm is shown in Figure 17a, for the case $a>b>0$; note how compact this is in comparison to the stencil of a standard second-order upwind scheme, shown in Figure 17b [27]. He also coined the name "N-scheme" for the first-order scheme that, on a cartesian grid, takes its data from the upwind triangle fitting the convection path most tightly ( $\mathrm{N}$ stands for narrow). For example, for point 1 in Figure 17a it would be triangle (124). This scheme, as shown in [5.3], is optimal in the sense that, among all schemes with upwind triangular domain of dependence, it combines the smallest truncation error with the largest stable time-step. The three-dimensional extension is also described in [53].

While the triangles in Sidilkover's work were still considered subdivisions of squares, they become autonomous in later work by other authors. A major step in the development of two-dimensional convection schemes was the realization that there are two types of triangles [54]: those with one inflow side and those with two intlow sides. This is illustrated in Figure 18. If there is only one inflow side, the fluctuation ap- 


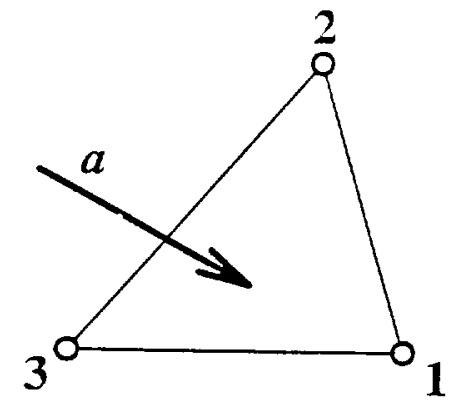

(a)

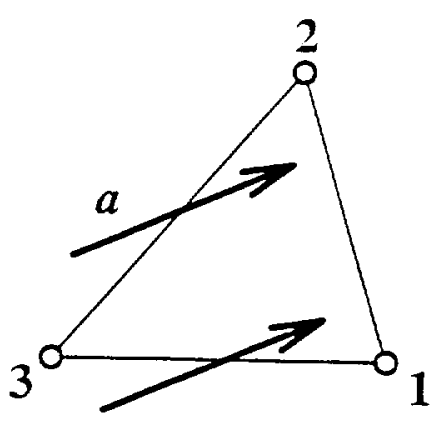

(b)

Figure 18: Two kinds of triangles: (a) with one inflow side; (b) with two inflow sides.

proach dictates that the entire residual be used to update the opposite node. This is the unique "single-target" form of the scheme, similar to the one-dimensional upwind scheme 2. If, however, there are two inflow sides, it may be argued that the residual be distributed over the two nodal points defining the third side. This is the "dualtarget" form of the particular scheme; each choice of distribution weights defines a new scheme. The spreading of the residual information over two points implies a potential loss of resolution, inherent to multi-dimensional numerical convection; there is no one-dimensional analogue of this effect.

In the development of multi-dimensional convection schemes, three design criteria play a decisive role. According to these, it is desirable for a scheme to be

1. linear: for a given grid geometry and flow angle the solution depends linearly on the data. This promotes convergence to a steady numerical solution. It is well known that the presence of nonlinear devices in the scheme, such as limiters [44] and frame rotation (see Section 4.2) can slow down or even halt the convergence process;

2. linearity preserving (LP): data of the form

$$
u(x, y)=b x-a y,
$$

which is a steady solution of Eq. 34 are not changed by the scheme. This promotes the accuracy of the scheme. It can be shown [54] that LP schemes yield second-order-accurate steady solutions of Eq. 34;

3. positive: the scheme has positive coefficients. This is sufficient for preventing numerical oscillations.

From one-dimensional finite-difference theory we know - and have known so for a long time - that the above conditions are mutually exclusive. There is a famous theorem by Godunov [24] which says that no linear convection-diffusion scheme with positive coefficients can be more than first-order accurate. With reference to our design criteria for multi-dimensional convection schemes this theorem reads:

There are no linear positive $L P$ schemes.

Again, nonlinearity is essential for the design of accurate, non-oscillatory schemes. 


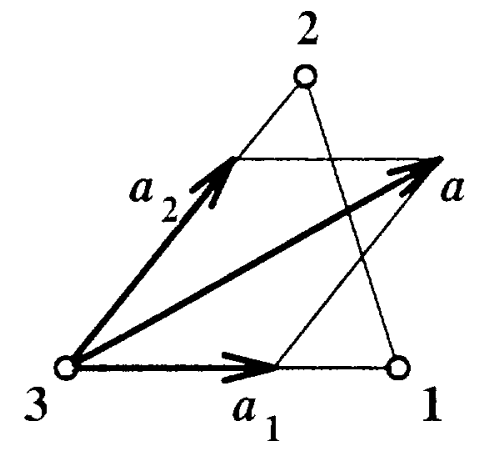

(a)

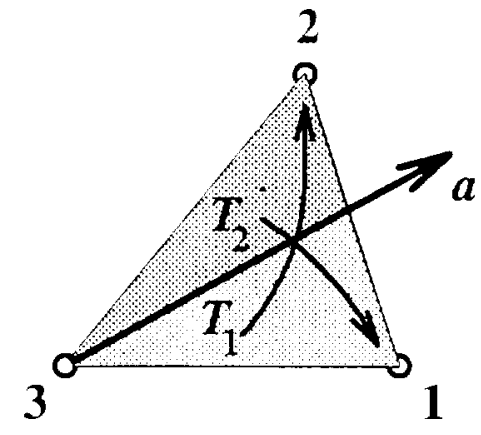

(b)

Figure 19: Dual-target form of convection schemes: (a) N-scheme; (b) LDA-scheme.

Among the various upwind convection schemes proposed in recent years, three schemes stand out; these are discussed below. They all are as compact as can be, requiring data on only one triangle for the approximation of the convection equation. A small miracle is that even positivity can be achieved uithout leaving the triangle. Of course, each nodal point is a vertex of a number of triangles and may receive fluctuations from several of these; programming therefore must be triangle-based. Some results of numerical experiments are presented in Section 6.

\section{The N-scheme: the optimal linear positive scheme}

The name of this scheme suggests equivalence to Sidilkover's N-scheme, but it actually is more general. Sidilkover's scheme is just the single-target form, common to all compact schemes; fluctuations from triangles requiring a dual-target scheme are ignored in the update. The dual-target form of the current $\mathrm{N}$-scheme uses distribution weights proportional to the components of the convection speed along the two inflow sides, as depicted in Figure 19a. This makes the scheme optimal in the sense of having the largest stability range for the time-step [54]. It is also linear and positive, and therefore can be no more than first-order accurate.

\section{The NN-scheme: the optimal nonlinear positive LP scheme}

This scheme is a nonlinear variant of the $\mathrm{N}$-scheme. hence the second $\mathrm{N}$. The nonlinear procedure included in this scheme has absolutely nothing in common with the TVDenforcing limiters included in one-dimensional convection schemes. It is based on the observation that in the convection equation (35) the component of the convection velocity $\vec{a}$ perpendicular to the solution gradient $\vec{\nabla} u$, has no effect on $u_{t}$. We therefore are allowed to replace $\vec{a}$ by any velocity that has the same component parallel to $\vec{\nabla} u$, as shown in Figure 20. This component, indicated by $\vec{a}_{w}$, is the velocity at which the level lines of $u$ normal normal to themselves, i.e. the wave speed of the local distribution of $u$. This wave speed is the smallest of all admissible convection speeds; it actually vanishes with the residual. We may now adopt the following strategy: if both $\vec{a}$ and $\vec{a}_{w}$ call for a dual-target scheme, we replace $\vec{a}$ by $\vec{a}_{w}$ in the N-scheme; in all other cases the scheme becones or remains a single-target scheme. In the case of 


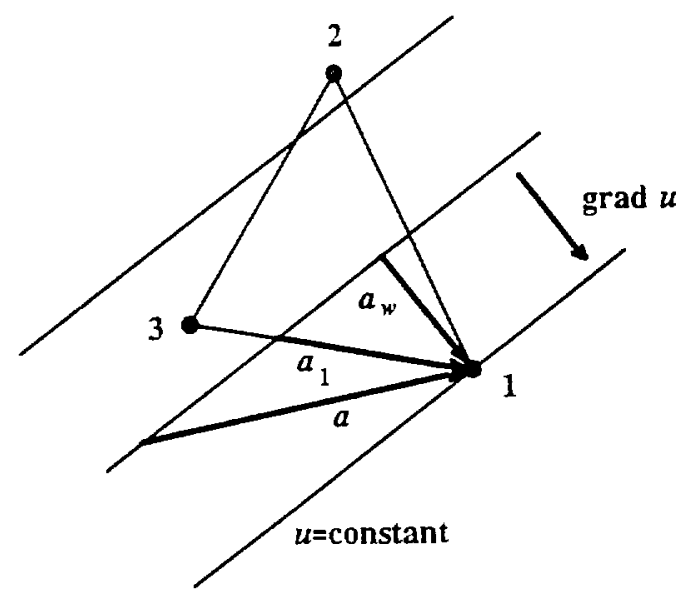

Figure 20: The NN-scheme: noulinear single-target form. The convection speed $\vec{a}$ calls for a dual-target scheme but is replaced by $\vec{a}_{1}$, which calls for a single-target scheme. The wave speed $\vec{a}_{w}$ is the component of $\vec{a}$ and $\vec{a}_{1}$ parallel to $\vec{\nabla} u$.

Figure 20, $\vec{a}$ is replaced by $\vec{a}_{1}$, the nearest admissible speed yielding a single-target scheme. The resulting scheme does not change any nodal value if the residual vanishes, hence is LP, and maximizes the allowable time step.

Numerical results indicate that the accuracy of the $\mathrm{NN}$-scheme lies between firstand second-order; see further Section 6 .

\section{The LDA scheme: a non-positive linear LP scheme}

This scheme is one of several low-diffusion schemes, designed for a low truncation error. In the dual-target form of the scheme the distribution weights are inversely proportional to the areas of the triangles cut from the mesh triangle by a streamline through the inflow vertex; see Figure 19b. This scheme is not positive, but very accurate: on a uniform grid it achieves third order accuracy, as demonstrated in Section 6.

The above schemes have served as the basis for convection-diffusion schemes in a study by Tomaich and Roe [55]. Since the diffusion operator can not be approximated on a single triangle, their schemes are formulated with reference to a central nodal point. Numerical solutions of the Simith-Hutton [56] test problem demonstrate that these schemes rival the best exsisting convection-diffusion schemes in accuracy. In addition, their way of discretizing the Laplacean is directly applicable to any of the disipative terms included in the Navier-Stokes equations; thus, the basis for genuinely multi-dimensional Navier-Stokes codes has been laid.

\section{Numerical results}

To support some of the statements made about the new, compact convection schemes I first show how these schemes fared in a comparative grid-refinement study by Jens Müller [57]. The problem is that of convection of a Gaussian distribution over a semicircle; Inflow is at $y=0, x<0$, outflow at $y=0, x>0$. Four kinds of grids were used, of which three examples are displayed in Figure 21. Grids $\alpha$ and $\beta$ derive from 
The $\alpha$-mesh

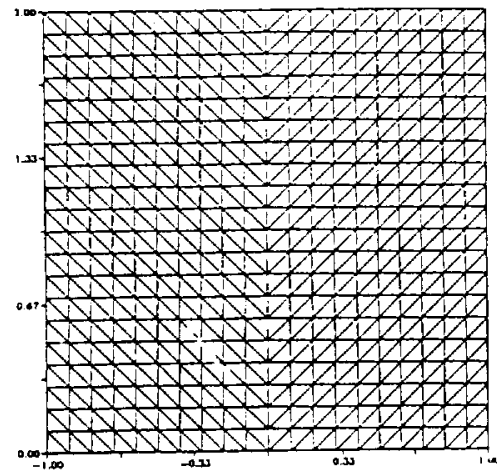

The $\beta$-mesh

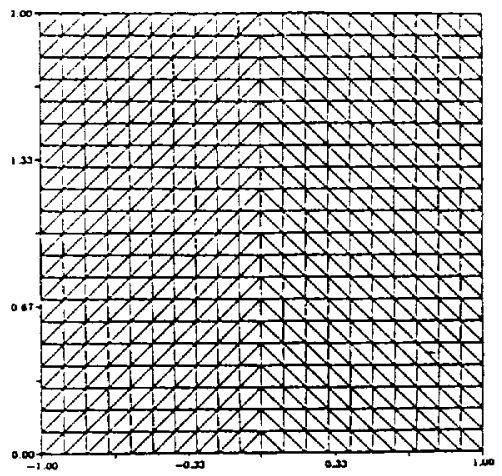

The $\gamma$-mesh

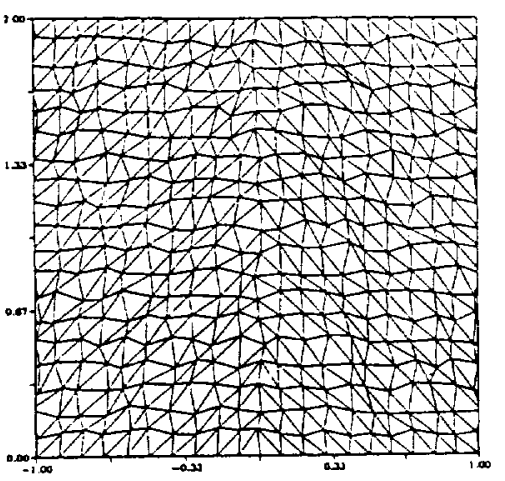

Figure 21: Three grids used in the circular-convection experiments.

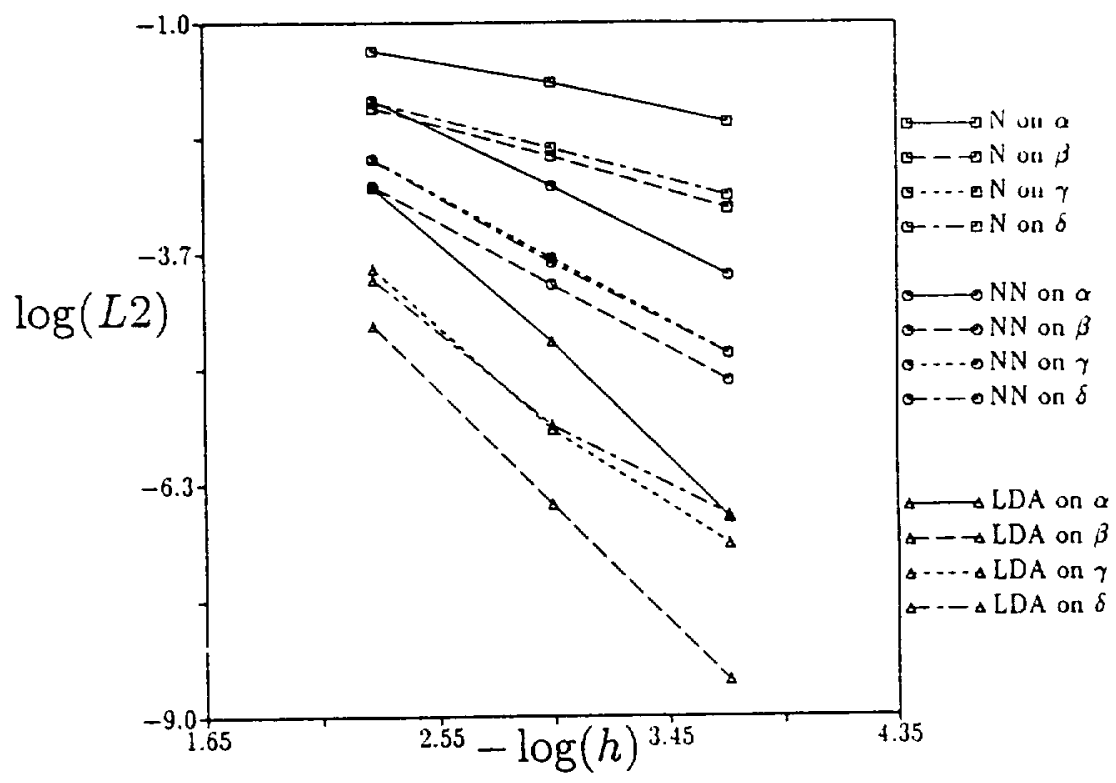

Figure 22: Grid-convergence of $L_{2}$-error for convection of a Gaussian over a semicircle by various schemes on various grids.

a uniform cartesian grid by adding diagonals. in grid $\alpha$ those diagonals are chosen that are least aligned with the convection direction, in $\beta$ those most aligned. Grid $\gamma$ is a irregular perturbation to $\beta$, while $\delta$ (not shown) is a minor perturbation to $\gamma$. Figure 22 shows the convergence of the $L_{2}$-error produced by the $N_{-}, N_{N}$ - and LDA-schemes on the different grids. From the slope of the graphs of $\log$ (error) versus $\log ($ mesh-width) the following conclusions can be drawn:

1. The N-scheme is somewhat less than first-order accurate;

2. The NN-scheme is closer to being second-order accurate than first-order accurate;

3. The LDA-scheme is third-order accurate on a regular grid, second-order or less on a perturbed grid; 

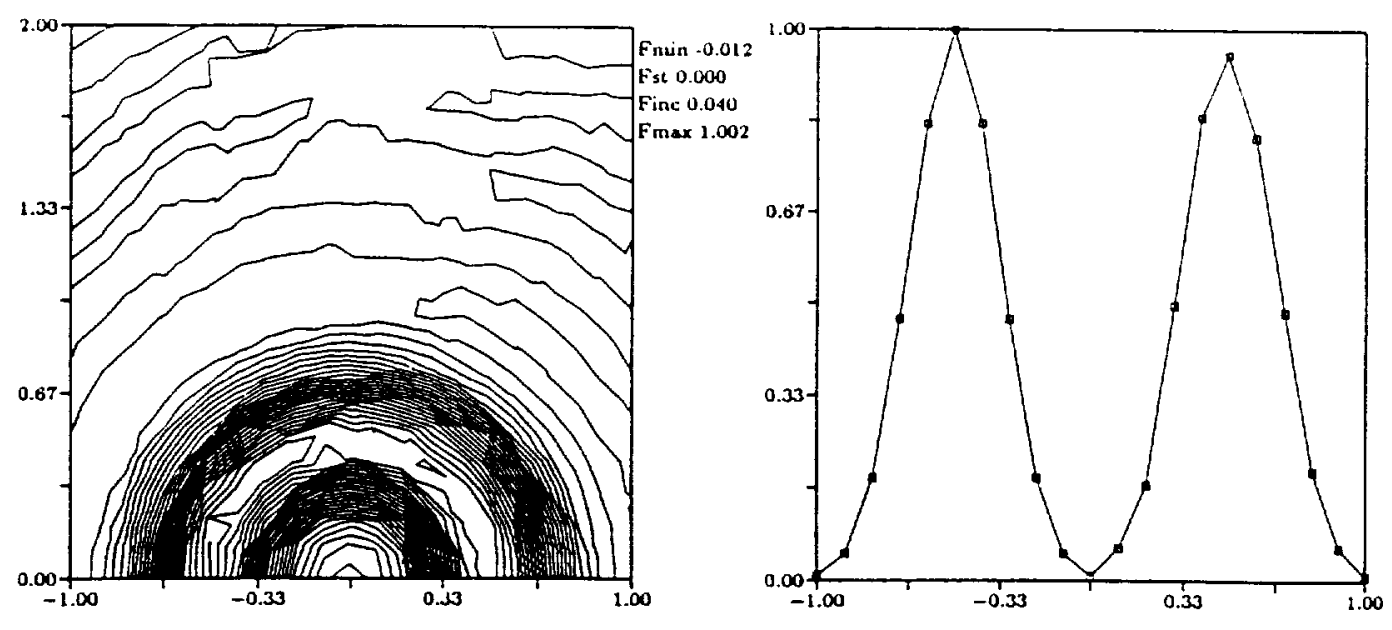

Figure 23: Contours (left) and cut along the $x$-axis (right) of the solution obtained with the LDA-scheme on a $20 \times 20 \beta$-grid.

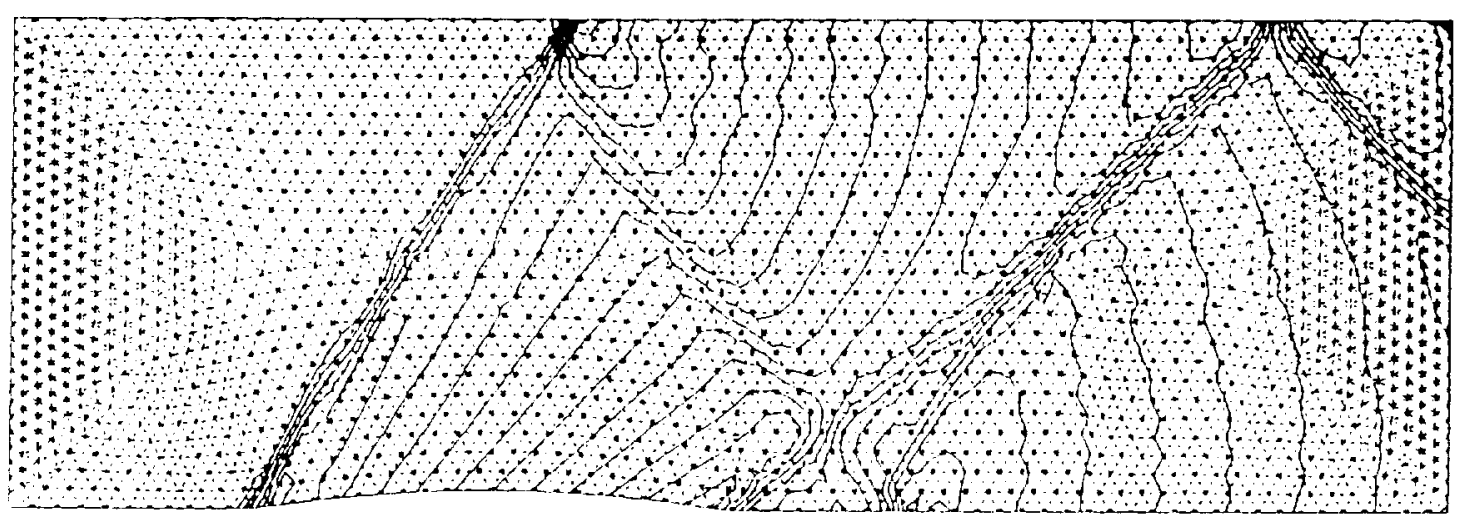

Figure 24: Inviscid flow at Mach 1.4 through an inlet, computed by a genuinely multi-dimensional upwind Euler code. Shown are Mach-number contours and the unstructured grid used.

4. All schemes decrease their error when diagonals are aligned with the flow.

Most surprising is the achievement of third-order accuracy on regular grids, considering the limited amount of information going into these compact schemes. Figure 23 gives an idea of this high accuracy by showing solution contours and a cut at $y=0$ obtained with the LDA-scheme on the very coarse $\beta$-grid of Figure 21 (Gaussian represented on 10 meshes). Similar results obtained with three-dimensional extensions of the schemes can be found in Deconinck's comprehensive review paper [4].

While the search for compact convection schemes continues, several authors are trying to put together the ingredients listed in Section 5, producing a genuinely multidimensional upwind Euler code. Advanced numerical results can be found in the present volume in the contribution by Catalano et al. An earlier successful calculation of supersonic inlet flow by Struijs et al. [3] produced the Mach-contours shown in Figure 24; superimposed is the moderately irregular triangulation. The results 


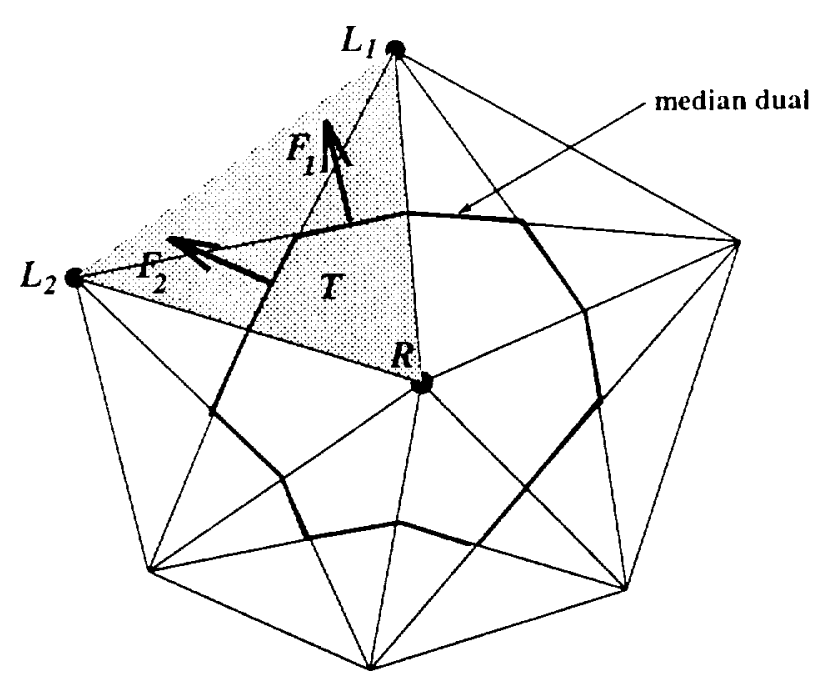

Figure 25: The finite-volume scheme of Barth, Powell and Parpia, based on a triangular grid and its median dual. The flux $F_{1}$ across one median element located in triangle $T$ is computed using wave data from triangle $T$ and fluxes from the vertices $L_{1}$ and $R$. For the flux $F_{2}$ across the other element in the same triangle the same wave data are used, but the fluxes are taken from $L_{2}$ and $R$.

demonstrate the excellent shock-capturing ability of the $\mathrm{NN}$-scheme.

\section{Finite-volume schemes revisited}

From Section 5 the reader might get the impression that genuinely multi-dimensional schemes can only be formulated on triangular and tetrahedral meshes, and that they are incompatible with the finite-volume formulation. If this were true, it would mean a serious restriction on the use of such schemes within the CFD community, for it is not at all clear that unstructured triangular or tetrahedral grids are the way of the future. An alternative, for instance, is offered by adaptive cartesian grids [58]. The emphasis on triangles in Section 5 arises from the experience that the numerical building blocks, e.g. Roe's matrix averaging, take their simplest form on such meshes. Therefore, in developing a multi-dimensional scheme of a different format it would be good practice to start with the wave decomposition of residuals on an underlying triangular grid.

As an example of such practice, consider the two-dimensional finite-volume Euler scheme of Powell, Barth and Parpia [59], illustrated in Figure 25. The wave model indeed is applied to data on triangular meshes; for the update, though, a finite-volume scheme is chosen. Cell faces are formed from the medians of the triangles, yielding the so-called median-dual grid. Across each median element of the cell contour a flux is computed using an equation of the form (26), where $L$ and $R$ denote the vertices of the triangle side bisected by the median, and the sum includes all waves identified in the triangle. Note the difference with the scheme of Rumsey et al., where the wave model would be based solely on $U_{R}-U_{L}$. The resulting nonlinear scheme, applied to a scalar convection equation, is LP and positive and appears to be more accurate than the $\mathrm{NN}$-scheme. 


\section{Conclusions}

The state of the art in genuinely multi-dimensional upwind differencing has made dramatic advances over the past three years, owing to a shift from the finite-volume approach to the flctuation approach. The basic ingredients for multi-dimensional Euler codes, i.e. wave model, conservation principle and convection scheme, are ready for integration, and the first numerical results look good. The coming years will yield many more Euler applications in two and three dimensions, further improvements in wave models and compact convection schemes, and extension of the approach to the modeling of the Navier-Stokes equations.

\section{Acknowledgement}

In preparing this lecture I received much-needed help from Jens Müller, Tim Tomaich, Phil Roe and Ken Powell, for which I thank them profoundly.

\section{References}

[1] P. L. Roe, "Upwind schemes using various formulations of the Euler equations," in Numerical Methods for the Euler equations of fluid dynamics, pp. 14-31, 1985.

[2] P. L. Roe, "Discrete models for the numerical analysis of time-dependent multidimensional gas-dynamics," Journal of Computational Physics, vol. 63, 1986.

[3] R. Struijs, H. Deconinck, P. DePalma, P. Roe, and K. Powell, "Progress on multidimensional Euler solvers on unstructured grids," in AIAA 10th Computational Fluid Dynamics Conference, 1991.

[4] H. Deconinck, R. Struijs, G. Bourgois, H. Paillère, and P. L. Roe, "Multidimensional upwind methods for unstruuctured grids," in Unstructured Grid Methods for Advection Dominated Flows, 1992.

[5] S. F. Davis, "A rotationally-biased upwind difference scheme for the E11ler equations," Journal of Computational Physics, vol. 56, 1984.

[6] D. Levy, K. G. Powell, and B. van Leer, "Implementation of a grid-independent upwind scheme for the Euler equations," in AIAA 9th Computational Fluid Dynamics Conference, 1989.

[7] D. Levy, Use of a Rotated Riemann Solver for the Two-Dimensional Euler Equations. PhD thesis, The University of Michigan, 1990.

[8] A. Dadone and B. Grossman, "A rotated upwind scheme for the Euler equations," AIAA Paper 91-06:35, 1991.

[9] A. Dadone and B. Grossman, "A rotated upwind scheme for the Euler equations," in Procedings of the 13 th International Conference on Numerical Methods in Fluid Dynamics, 1992. To appear. 
[10] S. Obayashi and P. M. Goorjian, "Improvements and applications of a streamwise upwind algorithm," in AIAA 9th Computational Fluid Dynamics Conference. 1989.

[11] Y. Tamura and K. Fujii, "A multi-dimensional upwind scheme for the Euler equations on unstructured grids," in Fourth International Symposium on Computational FLuid Dynamics, 1991.

[12] C. L. Rumsey, B. van Leer, and P. L. Roe, "A grid-independent approximate Riemann solver with applications to the Euler and Navier-Stokes equations," AIAA Paper AIAA 91-0239, 1991.

[13] C. L. Rumsey, B. van Leer, and P. L. Roe, "Effect of a multi-dimensional flux function on the monotonicity of Euler and Navier-Stokes computations," in AIAA 10th Computational Fluid Dynamics Conference, 1991.

[14] C. L. Rumsey, Development of a grid-independent approximate Riemann solver. PhD thesis, University of Michigan, 1991.

[15] C. L. Rumsey, B. van Leer, and P. L. Roe, "A grid-independent approximate Riemann solver for the Euler and Navier-Stokes equations," JCP, 1992. To appear.

[16] I. H. Parpia, "A planar oblique wave model for the Euler equations," in $A I A A$ 10th Computational Fluid Dynamics Conference, 1991.

[17] I. H. Parpia and D. J. Michalek, "A nearly-monotone genuinely multi-dimensional scheme for the Euler equations," AIAA Paper 92-035, 1992.

[18] T. J. Barth and P. O. Frederickson, "Higher order solution of the Euler equations on unstructured grids using quadratic reconstruction," AIAA Paper 90-0013, 1990.

[19] R. Abgrall, "Design of an essentially non-oscillatory reconstruction procedure on finite-element type meshes." ICASE Report 91-84, 1991.

[20] P. L. Roe, "Beyond the Riemann problem," in Algorithmic Trends in Computational Fluid Dynamics for the 90s, 1992.

[21] H. Deconinck, "Beyond the Riemann problem ii," in Algorithmic Trends in Computational Fluid Dynamics for the 90s, 1992.

[22] P. L. Roe, "Characteristic-based schemes for the Euler equations," Annual Review of Fluid Mechanics, vol. 18, pp. 337-365, 1986.

[23] P. L. Roe, "The use of the Riemann problem in finite-difference schemes," Lecture Notes in Physics, vol. 141, 1980.

[24] S. K. Godunov, "A finite-difference method for the numerical computation and discontinuous solutions of the equations of fluid dynamics," Matcmaticheshii Sbornik, vol. 47, pp. 271-306, 1959. 
[25] B. van Leer. "Towards the ultimate conservative difference scheme. IV. A new approach to mumerical convection," Journal of Computational Physics, vol. 23. 1977.

[26] B. van Leer, "Towards the ultimate conservative difference scheme. V. A secondorder sequel to Godunov's method," Journal of Computational Physics, vol. 32. 1979 .

[27] B. van Leer, "Upwind-difference methods for aerodynamic problems governed by the Euler equations," in Large-Scale Computations in Fluid Mechanics, Lectures in Applied Mathematics, vol. 22, 1985.

[28] P. L. Roe, "Fluctuations and signals, a framework for numerical evolution problems," in Numerical Methods in Fluid Dynamics, pp. 219-257, 1982.

[29] P. L. Roe, "Approximate Riemann solvers, parameter vectors and difference schemes," Journal of Computational Physics, vol. 43, 1981.

[30] S. Osher and F. Solomon, "Upwind schemes for hyperbolic systems of conservation laws," Mathematics and Computation, vol. 38, 1982.

[31] A. Harten, P. D. Lax, and B. van Leer, "Upstream differencing and Godunov-type schemes for hyperbolic conservation laws," SIAM Review, vol. 25, 1983.

[32] B. van Leer, A Choice of Difference Schemes for Ideal Compressible Flow. PhD thesis, Leiden University, 1970.

[33] B. van Leer, "Towards the ultimate conservative difference scheme. III. Upstreamcentered finite-difference schemes for ideal compressible flow," Journal of Computational Physics, vol. 2:3, 1977.

[34] B. van Leer, W. T. Lee, and K. C. Powell, "Sonic-point capturing," in AIAA 9th Computational Fluid Dynamics Conference, 1989.

[35] B. van Leer, W. T. Lee, and P. L. Roe, "Characteristic time-stepping or local preconditioning of the Euler equations," in AIAA 10th Computational Fluid Dynamics Conference, 1991.

[36] B. van Leer, "On the relation between the upwind-differencing schemes of Godunov, Engquist-Osher and Roe," SIAM Journal on Scientific and Statistical Computing, vol. 5, 1984.

[37] S. K. Godunov, A. W. Zabrodyn, and G. P. Prokopov, "A difference scheme for two-dimensional unsteady problems of gas dynamics and computation of flow with a detached shock wave," Zhur. Vych. Mat. i Mat. Fyz., vol. 1, p. 1020, 1961.

[38] P. R. Woodward and P. Colella, "The numerical simulation of two-dimensional fluid flow with strong shocks," Journal of Computational Physics, vol. 54, pp. 115-173, 1984.

[39] P. Colella and P. R. Woodward, "The piecewise-parabolic method (PPM) for gasdynamical simulations," Journal of Computational Physics, vol. 54, pp. 174-201, 1984. 
[40] S. K. Chakravarthy, A. Harten, and S. Osher. "Essentially non-uscillatory shockcapturing schemes of arbitrarily-high accuracy," AIAA Paper 86-0339. 1986.

[41] V. Venkatakrishnan, "Newton solution of inviscid and viscous problems," AIA.A Paper 88-0413, 1988.

[42] D. Levy, K. G. Powell, and B. van Leer, "Use of a rotated Riemann solver for the two-dimensional Euler equations," Journal of Computational Physics, 1991. Submitted.

[4:3] S. F. Davis, "Shock capturing." ICASE Report 85-25, 1985.

[44] W. K. Anderson, J. L. Thomas, and B. van Leer, "A comparison of finite volume flux vector splittings for the Euler equations," AIAA Journal, vol. 24, 1985.

[45] P. L. Roe and L. Beard, "An improved wave model for multidimensional upwinding of the Euler equations," in Proceedings of the 13th International Conference on Numerical Fluid Dynamics, 1992. To appear.

[46] L. A. Catalano, P. DePalma, and G. Pascazio, "A multidimensional solutionadaptive multigrid solver for the Euler equations," in Proceedings of the 13th International Conference on Numerical Methods in Fluid Dynamics, 1992. To appear.

[47] C. Hirsch, C. Lacor, and H. Deconinck, "Convection algorithm based on a diagonalization procedure for the multidimensional Euler equations," in $A I A A$ sth Computational Fluid Dynamics Conference, 1987.

[48] C. Hirsch and C. Lacor, "Upwind algorithms based on a diagonalization of the multidimensional Euler equations," AIAA Paper 89-1958, 1989.

[49] P. van Ransbeek, C. Lacor, and C. Hirsch, "A multidimensional cell-centered upwind algorithm based on a diagonalization of the Euler equations," in Proceedings of the 10th International Conference on Numerical Methods in Fluid Dynamics, 1990 .

[50] K. G. Powell and B. van Leer, "A genuinely multi-dimensional upwind cell-vertex scheme for the Euler equations," AIAA Paper 89-0095, 1989.

[51] P. L. Roe, R. Struijs, and H. Deconinck, "A conservative linearisation of the multidimensional Euler equations," Journal of Computational Physics, 1992. To appear.

[52] D. Sidilkover, Numerical Solution to Steady-State Problems with Discontinuities. $\mathrm{PhD}$ thesis, Weizmann Institute of Science, 1989.

[53) P. L. Roe and D. Sidilkover, "Optimum positive linear schemes for advection in two and three dimensions." Submitted to Journal of Computational Physics, 1991.

[54] H. Deconinck, R. Struijs, K. Powell, and P. Roe, "Multi-dimensional schemes for scalar advection," in AIAA 10th Computational Fluid Dynamics Conference. 1991. 
[5.5] (a. T. Tomaich and P. L. Roe, "Compact schemes for advection-diffusion schemes on unstructured grids." Presented at the 23 rd Annual Modeling and Simulation Conference. 1992.

[56] R. M. Smith and A. G. Hutton, "The numerical treatment of advection: A performance comparison of current methods," Numerical Heat Transfer, vol. 5. 1982.

[57] J.-D. Müller and P. L. Roe, "Experiments on the accuracy of some advection schemes on unstructured and partly structured grids." Presented at the $23 \mathrm{rd}$ Anuual Modeling and Simulation Conference. 1992.

[58] D. De Zeeuw and K. G. Powell, "An adaptively-refined carterian mesh solver for the Euler equations." To appear in Journal of Computational Physics, 1992.

[59] K. G. Powell, T. J. Barth, and I. F. Parpia, "A solution scheme for the Euler equations based on a multi-dimensional wave model." Extended abstract for the AIAA 31st Aerospace Sciences Meeting and Exhibit, 1992. 



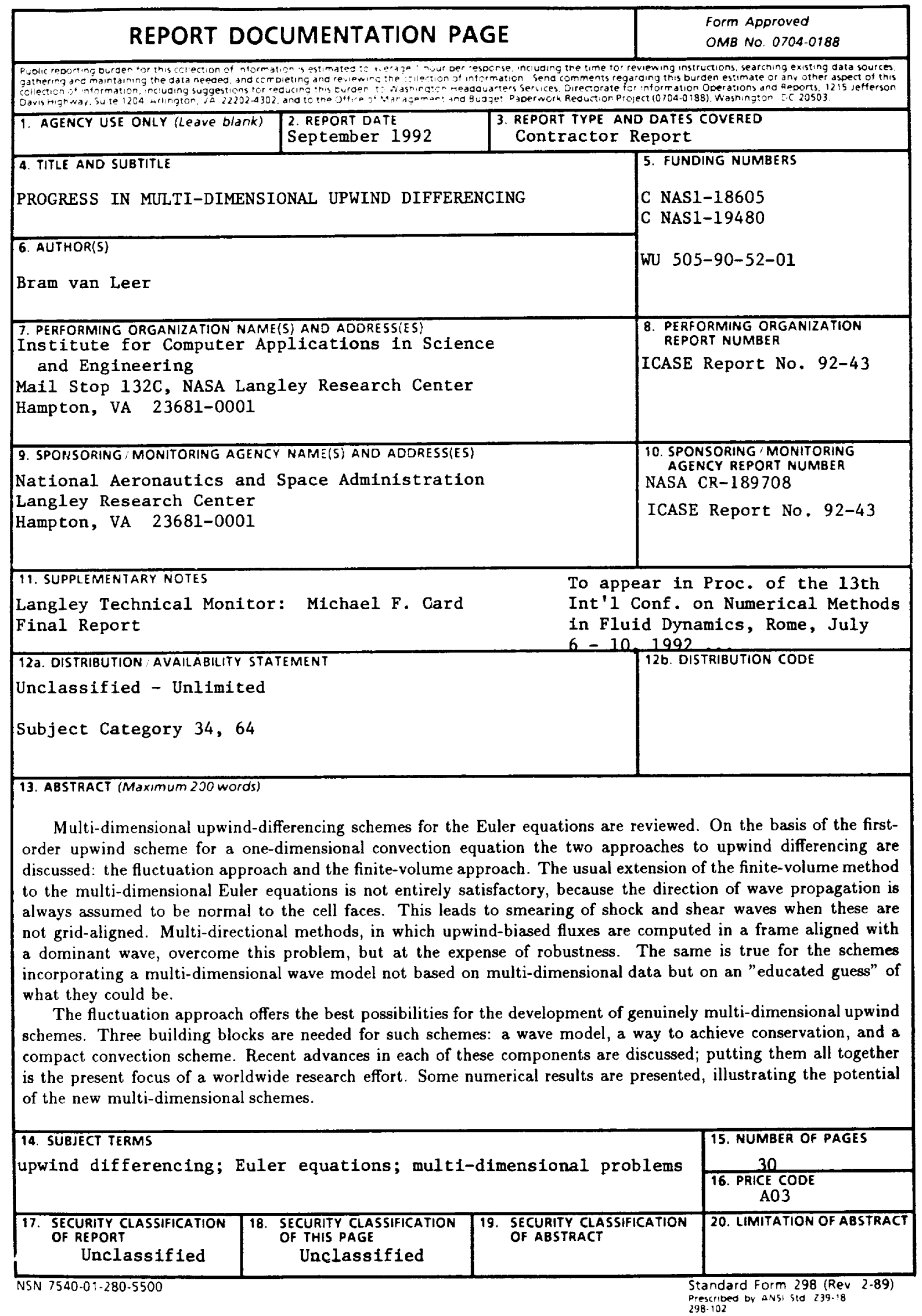

